\title{
Two-Dimensional Transition Metal Dichalcogenides and Their Charge Carrier Mobilities in Field-Effect Transistors
}

\author{
Sohail Ahmed ${ }^{1} \cdot$ Jiabao Yi $^{1}$
}

Received: 24 April 2017/Accepted: 11 July 2017/Published online: 16 August 2017

(C) The Author(s) 2017. This article is an open access publication

\begin{abstract}
Two-dimensional (2D) materials have attracted extensive interest due to their excellent electrical, thermal, mechanical, and optical properties. Graphene has been one of the most explored 2D materials. However, its zero band gap has limited its applications in electronic devices. Transition metal dichalcogenide (TMDC), another kind of 2D material, has a nonzero direct band gap (same charge carrier momentum in valence and conduction band) at monolayer state, promising for the efficient switching devices (e.g., field-effect transistors). This review mainly focuses on the recent advances in charge carrier mobility and the challenges to achieve high mobility in the electronic devices based on 2DTMDC materials and also includes an introduction of 2D materials along with the synthesis techniques. Finally, this review describes the possible methodology and future prospective to enhance the charge carrier mobility for electronic devices.
\end{abstract}

Keywords 2D materials $\cdot$ TMDC layers $\cdot$ Charge carrier mobility $\cdot$ Field-effect transistor $\cdot$ Heterostructure $\cdot$ Charge carrier scattering

\section{Introduction}

Discovery of graphene has diverted the interest of researchers toward a new family of nanomaterials known as $2 \mathrm{D}$ materials. Unique properties of $2 \mathrm{D}$ materials have been widely utilized for diverse applications such as catalysis, supercapacitors, energy storage devices, and high-performance sensors. Besides graphene, transition metal dichalcogenides (TMDCs) and layered oxide materials are also parts of 2D materials family. 2D materials have shown promising properties in the application of electronic devices $[1,2]$. Semiconductor behavior of 2D materials (e.g., $\mathrm{MoS}_{2}$ ) makes them promising materials for field-effect transistors (FETs). The FET fabricated from 2D materials will not only exhibit improved performance, in

Jiabao Yi

jiabao.yi@unsw.edu.au

1 School of Materials Science and Engineering, UNSW, Kensington, Sydney 2052, Australia terms of fast processing rate and low power consumption, but also allow further reduction in device dimensions which is the need for the fabrication of next-generation electronic devices. In recent years, many efforts have been made to review the research on the synthesis, characterization of single- and few-layered 2D materials including their electronic, magnetic, optical and mechanical properties as well as applications [3-9]. Furthermore, some reviews focused on the utilization of $2 \mathrm{D}$ materials in a variety of applications such as flexible and transparent electronic, optoelectronic devices [10-15], energy conversion and storage [16, 17], hydrogen generation [18], and gas sensors [19]. It is well known that most 2D layered materials exist in a bulk state. These materials have layered structure and weak interlayer van der Waals force holding these layers together. Layered structure makes it possible to achieve monolayer or a few layers of $2 \mathrm{D}$ materials by mechanical exfoliation. On the other hand, in-plane atoms are connected with strong covalent bonds $[1,20]$. Hence, monolayer 2D materials possess two-dimensional features 
in the lateral $\mathrm{x}-\mathrm{y}$ direction and quantum confinement in the third dimension, which makes these materials unique from their bulk counterparts. One of the features is high carrier mobility, which is essential for high-speed transistors. For a high-performance transistor, good ohmic contact, higher carrier mobility, and appropriate band gap $(\sim 1 \mathrm{eV})$ are the basic requirements [12]. Graphene, the most extensively studied 2D material, has exhibited very high carrier mobility $\left(\sim 2 \times 10^{5} \mathrm{~cm}^{2} \mathrm{~V}^{-1} \mathrm{~s}^{-1}\right)$ at the temperature of $5 \mathrm{~K}$ [21]. However, it is not suitable for logic applications due to its zero band gap, resulting in very small on/off ratio $(<10)$ at ambient temperature. In order to improve its on/ off ratio, the opening of band gap has been proposed. However, engineering the band gap up to $\sim 400 \mathrm{meV}$ will lead to a decrease in the mobility to less than $200 \mathrm{~cm}^{2}$ $\mathrm{V}^{-1} \mathrm{~s}^{-1}$. Similarly, p-type device, based on the graphene nanoribbons, demonstrates a band gap opening due to the various edge (armchair or zigzag) structures resulting in very high on/off ratio of $\sim 10^{6}$ and extremely low charge carrier mobility of $\sim 100-200 \mathrm{~cm}^{2} \mathrm{~V}^{-1} \mathrm{~s}^{-1}$, as compared to other members of graphene family. However, these devices have shown the subthreshold slope (SS) of $\sim 210 \mathrm{mV}$ per decade, which is not desirable and ideal. It needs to be $\sim 60 \mathrm{mV}$ per decade at room temperature [22]. In contrast to graphene, TMDCs, such as $\mathrm{MoS}_{2}(1.8 \mathrm{eV}), \mathrm{WS}_{2}(2.1 \mathrm{eV}), \mathrm{MoTe}_{2}$ $(1.1 \mathrm{eV})$, and $\mathrm{WSe}_{2}(1.7 \mathrm{eV})$, have desirable band gap $[12,23,24] . \mathrm{MoS}_{2}$ is one of the most promising materials for logic devices such as metal-oxide-semiconductor field-effect transistors (MOSFETs) due to its tunable band gap, high on/off ratio and relatively cheap price. The band gap of monolayer $\mathrm{MoS}_{2}$ has been reported to be $1.8 \mathrm{eV}$ earlier. However, recent work demonstrates it has a direct band gap of $2.5 \mathrm{eV}$, while its bulk counterpart has an indirect band gap of $1.2 \mathrm{eV}$ [25-28]. Being a relatively large band gap material, experimentally, $\mathrm{MoS}_{2}$ only shows low carrier mobility of $\sim 1 \mathrm{~cm}^{2} \mathrm{~V}^{-1} \mathrm{~s}^{-1}$ without high- $k$ dielectric gate material [29], whereas the mobility can reach $\sim 150 \mathrm{~cm}^{2} \mathrm{~V}^{-1} \mathrm{~s}^{-1}$ at $300 \mathrm{~K}$ with $\mathrm{HfO}_{2}$ as the top-gate layer [30-33]. In addition, theoretical calculation based on density functional theory has indicated that the mobility of $\mathrm{MoS}_{2}$ can reach $400 \mathrm{~cm}^{2} \mathrm{~V}^{-1} \mathrm{~s}^{-1}$ [34] at room temperature. More recently, another type of 2D material, black phosphorus, has been predicted to have a hole mobility of $10,000-26,000 \mathrm{~cm}^{2}$ $\mathrm{V}^{-1} \mathrm{~s}^{-1}$ [35], and experimentally, a mobility of $1000 \mathrm{~cm}^{2} \mathrm{~V}^{-1} \mathrm{~s}^{-1}$ has been achieved [36], showing very promising potential for electronic devices [37].

In this review, we mainly focus on the recent development of the charge carrier mobility in 2D TMDC materials and the challenges for achieving high mobility as well as high current on/off ratio simultaneously, which are essential for 2D TMDC-based electronic devices. We also give an introduction of 2D materials (including TMDCs) and their synthesis using different approaches. Finally, this review describes the possible methodology and future prospective to enhance the charge carrier mobility for electronic devices.

\section{2D Materials}

Recent advancements in science and technology have unveiled the new prospects and put the mankind on the foundation of the newly developed field named as nanotechnology. This technology has enabled us to conduct research and work in the domain up to nanometer scale, resulting in the technologies which were never possible earlier. These technologies include cancer therapy based on nanoparticles, nanocomposites and innovative medicine, high-performance nanoelectronics, and highly sensitive sensors [38-41]. Evolution of nanotechnology has also introduced the distinguished class of low-dimensional systems such as zero-dimensional (OD, i.e., nanoparticles), one-dimensional (1D, i.e., nanowires), two-dimensional (2D, i.e., graphene), and three-dimensional (3D, i.e., bulk materials). The low-dimensional system plays an important role in classifying the nanomaterials, as the dimension of the material will not only define the atomic structure but also the properties of the nanomaterials [42].

In 2004, Geim and Novoselov [43] obtained a layered structure by mechanical exfoliation using scotch tape, which is later called graphene. The discovery of graphene has opened a new area of research-2D materials. These materials have shown many excellent properties widely used for energy, sensors, catalysis, electronic devices, spintronic devices as well as biomedical applications [3]. The discovery of graphene has triggered the research interest toward other two-dimensional materials, such as silicene, black phosphorus, transition metal dichalcogenides (TMDCs), and layered oxide materials. One of the most important features of these 2D materials is the high mobility of the carriers due to their quantum confinement in the third dimension, which is promising for the applications of electronic devices, such as transistors [1, 2].

As described previously, graphene has exhibited very high carrier mobility [21]. However, it is unsuitable for the applications in transistors since materials used to make transistors have to be able to switch current on and off to create logic circuits. Different from graphene, $\mathrm{MoS}_{2}$, one of the TMDCs, has a direct band gap in monolayer structure, which makes it possible to tune the carriers transport in an electronic device, thus realizing the device functions. Among the 2D materials, currently, TMDCs have attracted more and more interest due to their natural abundance and unique/diverse properties. The generalized chemical formula for TMDCs is $\mathrm{MX}_{2}$, where $\mathrm{M}$ represents the transition metal (typically $\mathrm{Ti}, \mathrm{Zr}, \mathrm{Hf}, \mathrm{Mo}, \mathrm{W}, \mathrm{Nb}, \mathrm{Re}, \mathrm{V}$, and Ni) 
Table 1 2D materials family [1, 9, 20, 29, 42, 66-70]
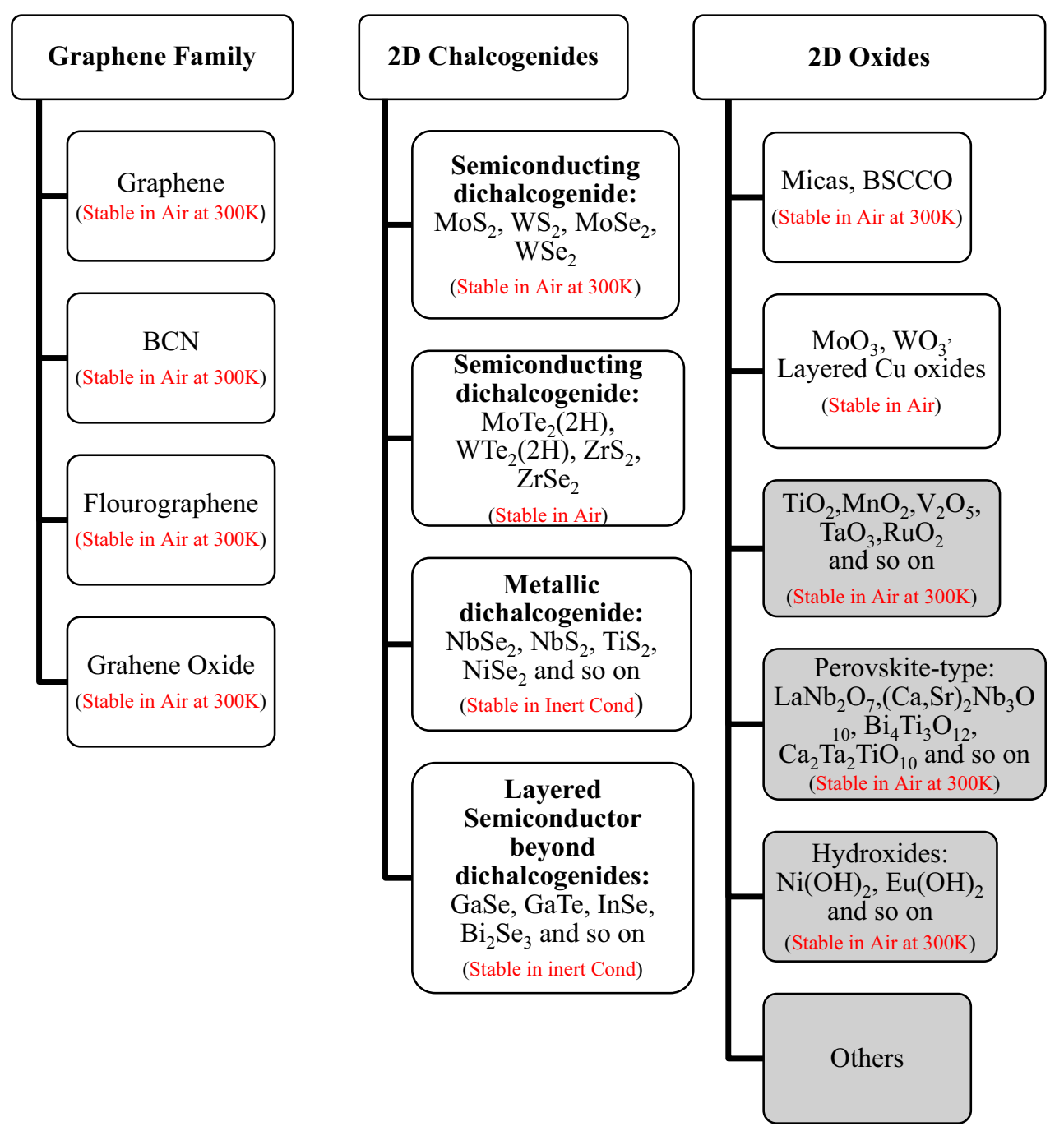

of group 4-10 [26, 44] and X is a chalcogen (S, Te, and Se) [45]. Presently, more than 40 different TMDCs combinations have been reported [28, 46-48] and they have shown distinctive properties. In TMDCs, $M$ (transition metal) layer sandwiched between two (02) X (chalcogen) atomic layers. Different atomic arrangements can generate the octahedral (tetragonal, T) and trigonal prismatic (hexagonal, H) structure of the 2D TMDCs. In H-phase TMDC, hexagonal symmetry can be observed from a top view and $\mathrm{X}-\mathrm{M}-\mathrm{X}$ arrangement is considered to be the monolayer, in which each $\mathrm{M}$ atom is covalently bonded to the six $\mathrm{X}$ atoms [49], whereas T-phase has a trigonal arrangement of the chalcogen (X) atoms on the top and shows the hexagonal structure of chalcogen atoms from a top view [50]. Among both, the $2 \mathrm{H}$ phase of $2 \mathrm{D}$ TMDCs is stable in air [51].The stability of mono or few layer TMDC is of the vital importance due to its layer dependent properties [52]. The

conductivity of $1 \mathrm{~T} \mathrm{MoS}$ phase is $10^{7}$ times better than $2 \mathrm{H}$ $\mathrm{MoS}_{2}$ phase [53]. Due to the tunable band gap, quantum confinement and surface effects, monolayer 2D TMDCs $\left(\mathrm{MoS}_{2}, \mathrm{WS}_{2}\right)$ exhibit strong photoluminescence (PL) and large exciton binding energy [54]. Besides the TMDCs, two-dimensional oxides have also been extensively investigated. Two-dimensional oxides include micas and layered oxides, such as $\mathrm{MoO}_{3}$ [55] and $\mathrm{WO}_{3}$ (micas) [56], $\mathrm{TiO}_{2}$, $\mathrm{MnO}_{2}, \mathrm{~V}_{2} \mathrm{O}_{5}, \mathrm{TaO}_{3}$, and $\mathrm{RuO}_{2}$ [28, 57-61]. These oxides have also shown very promising properties for a variety of applications.

The family members of 2D graphene, TMDCs, and oxide materials are shown in Table 1 . Gray color shows the monolayer fabricated by exfoliation. These 2D materials can be categorized in several ways in terms of their electrical, mechanical and transport properties, as they possess excellent and unique mechanical, thermal, optical, and 
electronic properties [62-65] which have the potential to replace current silicon-based semiconductor devices in the future.

\subsection{Classification of 2D Materials}

For a better understanding of properties and its applications, 2D materials can be categorized in following three classes.

\subsubsection{Layered van der Waals Solids}

The most common type of 2D material is the layered van der Waals solid which has strong in-plane covalent or ionic bond and weak interlayer van der Waals bonding. This weak out-of-plane bonding allows the extraction of mono or few layers of 2D materials from their bulk counterpart through mechanical or liquid exfoliation. The dimensions of these materials in the lateral direction are up to a few micrometers and are less than $1 \mathrm{~nm}$ in thickness. TMDCs, especially $\mathrm{MoS}_{2}, \mathrm{MoSe}_{2}$, and $\mathrm{WS}_{2}$, are the well-studied materials. Besides those materials described afore, presently there are more than 40 different combinations of $2 \mathrm{D}$ layered TMDCs (X-M-X) reported [46, 47]. The transition metal, presenting in TMDCs, occupies trigonal prismatic or octahedral coordinates and forms the hexagonal structure [71]. Besides TMDCs, there are some other members of layered van der Waals solids as well, such as $\mathrm{Sb}_{2} \mathrm{Te}_{3}$ [72], vanadium oxide [73], and h-BN [74] etc.

\subsubsection{Layered Ionic Solids}

In this type of 2D materials, charged polyhedral layer is present between two layers of halide or hydroxide layers and these layers are held together via electrostatic force between them. Ion exchange liquid exfoliation or ion intercalation can be used to exfoliate the mono or few layered 2D materials. Typical layered ionic materials exfoliated from ion exchange methods are $\mathrm{KCa}_{2} \mathrm{Nb}_{3} \mathrm{O}_{10}$ [75], $\mathrm{RbLnTa}_{2} \mathrm{O}_{7}, \mathrm{~K}_{2} \mathrm{Ln}_{2} \mathrm{Ti}_{3} \mathrm{O}_{10}$ [75], and $\mathrm{La}_{0.9} \mathrm{Eu}_{0.05} \mathrm{Nb}_{2}$ $\mathrm{O}_{7}[76]$.

\subsubsection{Surface-Assisted Non-Layered Solids}

This type of 2D nanostructure materials is synthesized by making the layers artificially stacked on a substrate with arbitrary angles [77]. The methods to synthesize these materials include epitaxial growth and chemical vapor deposition. Silicene is a typical example of this class. However, its instability at ambient condition is the real challenge to make it feasible for the application in electronic applications [78, 79]. $\mathrm{Ge} / \mathrm{Ag}(100)$ and $\mathrm{Ag}(111)$ [80, 81], $\mathrm{TiO}_{2}$ [82] and $\mathrm{MgO} / \mathrm{Mo}$ (001) [83], and $\mathrm{Al}_{2} \mathrm{O}_{3}$ /
$\mathrm{SiO}_{2}[84]$ are the examples of surface-assisted non-layered solids.

\subsection{Synthesis Techniques for 2D Materials}

2D materials can be synthesized by following techniques:

\subsubsection{Micromechanical Exfoliation}

This process was first discovered by Geim and Novoselov in 2004 [43], and the monolayer graphene was achieved [85], in which parent bulk material was peeled off by micromechanical force of the scotch tape and placed on the surface of the photoresist. This cleavage is possible due to the weak interlayer van der Waals forces. Monolayers of $\mathrm{NbSe}_{2}$ [29], $\mathrm{MoS}_{2}[29,86]$, and $\mathrm{WS}_{2}$ [87] are some of the examples obtained by this process besides graphene. Despite being the fast and cost-effective process, micromechanical exfoliation is not an industrial-level production of monolayer materials since most of the flakes are smaller than $20 \mu \mathrm{m}$ in diameter. In addition to the monolayer, a few layers or very thick layers can also be achieved at the same time. On the other hand, because of the absence of chemical interaction, monolayer obtained by this process is highly crystalline and its structural integrity can be maintained. This monolayer has shown good stability at ambient conditions up to the months' exposure $[29,88]$.

\subsubsection{Liquid Exfoliation}

In contrast to mechanical exfoliation, which is a low yield method, liquid exfoliation is capable of producing the mono or few layers of $2 \mathrm{D}$ materials at a large scale. Mono or few layers of $\mathrm{Bi}_{2} \mathrm{Te}_{3}, \mathrm{TaSe}_{2}, \mathrm{MoSe}_{2}, \mathrm{MoTe}_{2}, \mathrm{BN}, \mathrm{WS}_{2}$, and $\mathrm{MoS}_{2}$ can be easily obtained by this method [1, 89-91]. This method can be categorized into four different forms such as oxidation, intercalation, ion exchange, and ultrasonic cleavage.

Graphene oxide can be synthesized with an oxidation method by treating graphite flakes with potassium permanganate or potassium chlorate and nitric acid or sulfuric acid or their mixture [92-94]. The addition and dispersion of epoxide functional groups or $-\mathrm{OH}$ in a polar solvent and subsequent sonication result in exfoliated graphene oxide. This method is also known as Hummers method or modified Hummer method [94, 95]. An oxidative form of liquid exfoliation is most suitable for layered materials possessing low reduction potential.

The intercalation technique is equally applicable to TMDCs and graphene. In this method, interlayer force decreases due to the intercalation of ionic or organic 
(a)

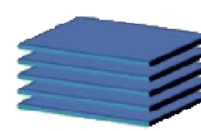

\section{Intercalation $\downarrow$}

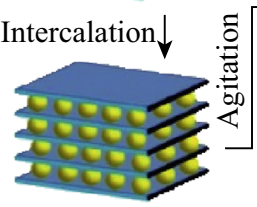

(c) (b)

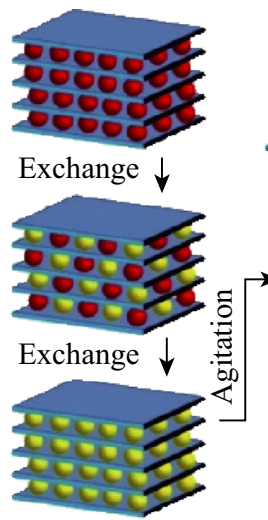

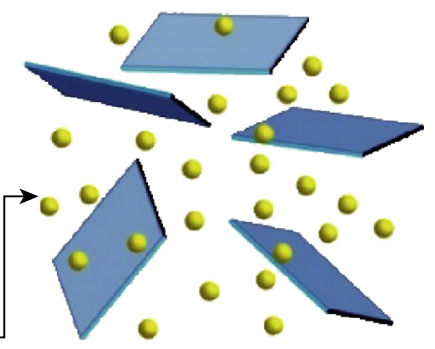
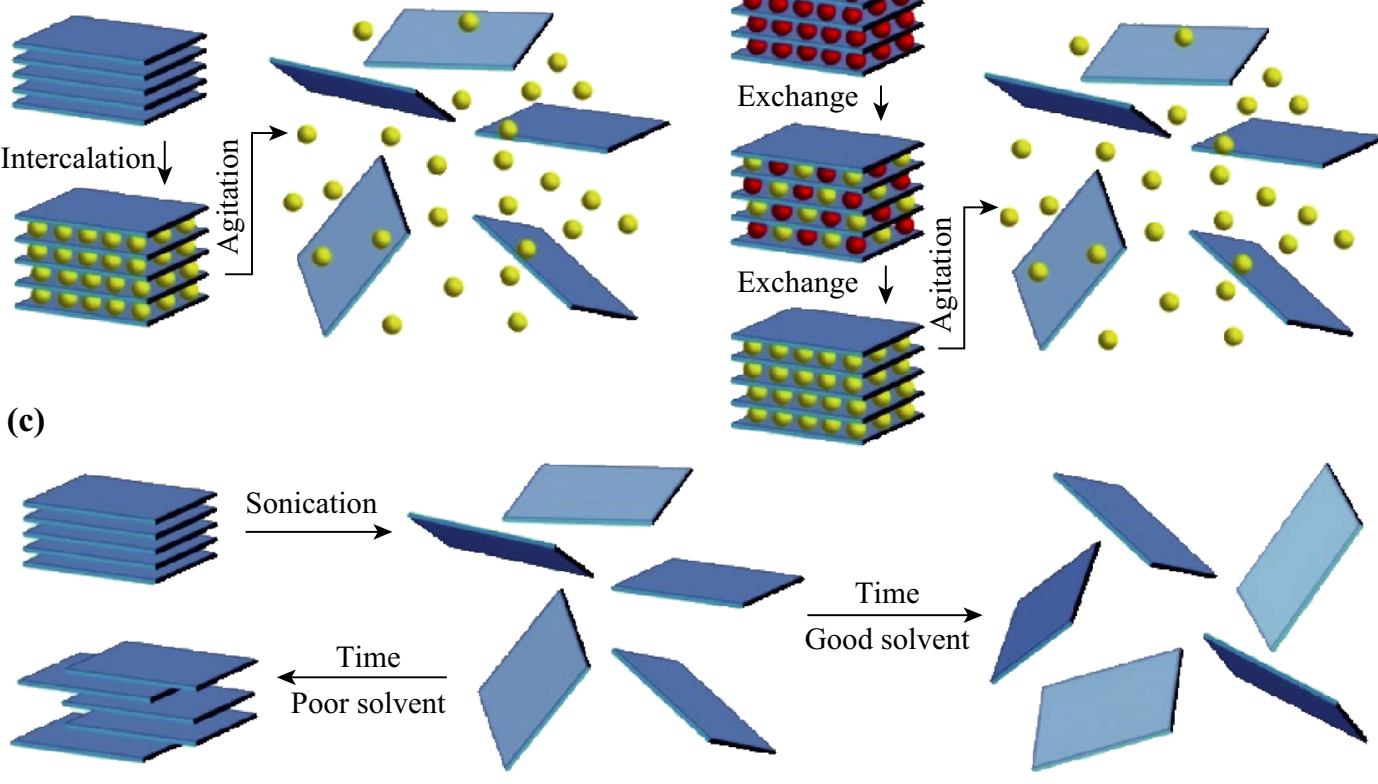

(d)

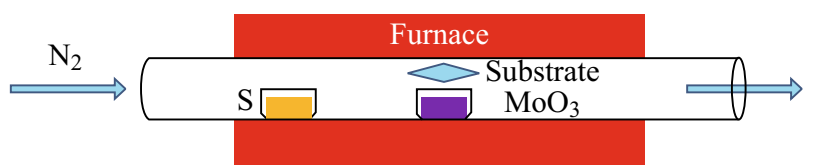

(e)
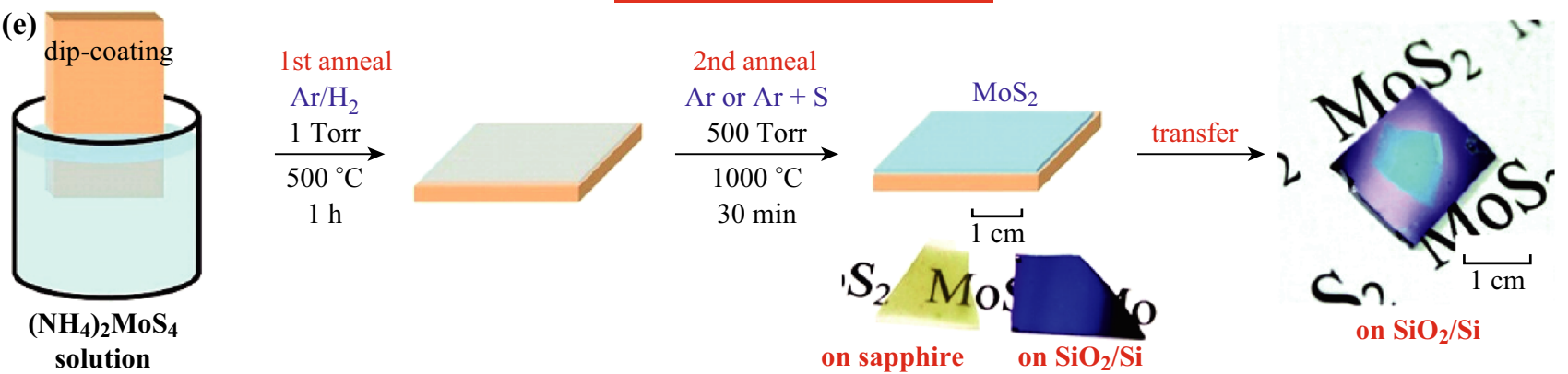

Fig. 1 Schematic representation of the variation in liquid exfoliation process: a intercalation, $\mathbf{b}$ ion exchange, and $\mathbf{c}$ ultrasonic exfoliation. ac Reprinted from Ref. [98] with permission from Copyright 2013, AAAS. d Schematic representation of the CVD process. e Schematic representation of large-area $\mathrm{MoS}_{2}$ nanosheet synthesis via dip and anneal technique. Reprinted with permission from Ref. [101], Copyright 2012, American Chemical Society

molecules, which results in a decrease in energy required for exfoliation [96, 97].

Another technique to obtain a single- or few-layered 2D material by liquid exfoliation is ultrasonic cleavage. In this technique, parent bulk material consisting of layers with weak interlayer bonding is ultrasonicated for $1-3 \mathrm{~h}$ after dispersion in a suitable solvent. In this process, cavitation bubbles are developed in the solvent due to high-energy ultrasonic waves [98]. The exfoliation of layers is possible due to the pressure released by the burst of cavitation bubbles. Subsequently, centrifugation is applied to separate the crystals. Figure 1a-c represents the schematic diagram of the above-mentioned forms of liquid exfoliation. Due to the complexity and destructive nature of liquid exfoliation method, layered 2D nanostructure material cleaved by mechanical exfoliation remains the favorite choice in the research community [9].

\subsubsection{Chemical Vapor Deposition (CVD)}

CVD is known as a bottom-up technique allowing the synthesis of 2D layers at a large scale with uniform thickness, which is promising for optoelectronics and electronic devices. Synthesis of $\mathrm{MoS}_{2} 2 \mathrm{D}$ nanostructured sheets by CVD on an oxidized silicon substrate is one of the recent developments. Generally, there are two ways for the fabrication of monolayer 2D materials. $\mathrm{MoS}_{2}$ as an example, first, in a so-called two-step bottom-up technique, 
a thin layer of metallic Mo is evaporated by an e-beam evaporation system. The Mo layer then reacts with sulfur vapors, generated by heating the elemental sulfur above its melting temperature. This reaction occurs at $750{ }^{\circ} \mathrm{C}$ leading to the formation of monolayered $\mathrm{MoS}_{2}$ [99]. This method can yield $\mathrm{MoS}_{2}$ with a thickness more than three layers. Second, a so-called one-step bottom-up technique is used for the synthesis of the atomically thick $\mathrm{MoS}_{2}$ nanosheets. In this approach, pure $\mathrm{S}$ and $\mathrm{MoO}_{3}$ powders are put in a CVD system. The mixture is heated to $650{ }^{\circ} \mathrm{C}$. Then, the atomic layer of $\mathrm{MoS}_{2}$ will be grown on the $\mathrm{Si}$ substrate, which is covered with the rGO (reduced graphene oxide) for $\mathrm{MoS}_{2}$ layers growth. The rGO acts as a catalyst and the seed to enhance the growth of $\mathrm{MoS}_{2}$ layers [100], as shown in Fig. 1d. By this approach, $\mathrm{MoS}_{2}$ monolayer with a thickness of $0.72 \mathrm{~nm}$ has been obtained. Furthermore, large-area $\mathrm{MoS}_{2}$ nanostructured sheets can be obtained by dip coating of $\left(\mathrm{NH}_{4}\right)_{2} \mathrm{MoS}_{4}$ (ammonium thiomolybdate) dissolved in DMF (dimethylformamide) on silicon or silicon dioxide substrate [100, 101]. Figure 1e shows the pictorial representation of this approach. After the $\left(\mathrm{NH}_{4}\right)_{2} \mathrm{MoS}_{4}$ solution is dipped onto the substrate, the substrate is annealed under $\mathrm{Ar} / \mathrm{H}_{2}$ atmosphere at $500{ }^{\circ} \mathrm{C}$ for $1 \mathrm{~h}$. Then, the substrate is subsequently performed the second annealing at $1000{ }^{\circ} \mathrm{C}$ for 30 min under $\mathrm{Ar}$ or $\mathrm{Ar}^{+} \mathrm{S}$ atmosphere [101]. $\mathrm{MoS}_{2}$ 2D layer nanosheets are then obtained. X-ray diffraction (XRD), atomic force microscope (AFM), and transmission electron microscopy (TEM) analysis indicate that the layers grow and overlap each other without interlayer chemical bonding. Besides $\mathrm{MoS}_{2}$, other 2D materials such as $\mathrm{TiS}_{2}$ [102], VSe ${ }_{2}$ [103], $\mathrm{WSe}_{2}$ [104], $\mathrm{WS}_{2}$ [105], and $\mathrm{MoSe}_{2}$ [106] were also produced using CVD technique.

In addition, thermally decomposed $(\mathrm{BN})_{3} \mathrm{H}_{6}$ (borazine) or $(\mathrm{ClBNH})_{3}$ B-trichloroborazine can be used to fabricate the layered $\mathrm{BN}$ on transferred metal surfaces, e.g., $\mathrm{Rh}, \mathrm{Pd}$, $\mathrm{Ru}, \mathrm{Ni}$, and $\mathrm{Pt}$, through an ultrahigh-vacuum CVD technique at a temperature above $700{ }^{\circ} \mathrm{C}$ [107-109].

\subsubsection{Van der Waals Epitaxial Growth on a Substrate}

This technique is similar to the CVD method. The only difference is that the substrate used in van der Waals epitaxial method also acts as a seed crystal. A variety of layered 2D nanostructured sheets can be synthesized by this method, such as $\mathrm{MoS}_{2}$, GaSe, h-BN, and hexagonal Si [107, 110-112].

Hexagonal $\mathrm{Si}$ is one of the examples achieved by this method. Si superstructures were deposited on Ag (001) substrate by heating the Si single crystal in ultrahigh vacuum using direct current. However, low-energy electron diffraction (LEED), scanning tunneling microscopy (STM), and XRD analysis indicated that there are two kinds of superstructures in the deposited layer [113]. Initially, a monolayer of $\mathrm{Si}$ nanostructure $\mathrm{p}(3 \times 3)$ was formed. Subsequently, complex structure $p(7 \times 4)$ was observed. Direct current deposition formed the atomic thick Si nanoribbons on Ag (110) substrate. The atoms in $\mathrm{Si}$ nanoribbons were arranged in a honeycomb structure [114, 115]. Minor distortion was produced by the Si nanostructure on Ag substrate. Similar to that of hexagonal $\mathrm{Si}$, monolayered Ge was also obtained by van der Waals epitaxial growth method on Ag (110) and Ag (111) substrates [116], whereas, due to the high solubility of Ge in $\mathrm{Ag}$, the tetramers structure of Ge on Ag substrate leads to a larger distortion than that of Si [116].

Large-area 2D nanostructured materials, which are difficult to obtain via liquid and mechanical exfoliation, may be synthesized by van der Waals epitaxial growth method. However, the structure and properties of the 2D materials fabricated by this method are critically dependent on the orientation of the substrate and its chemistry. Moreover, in respect of future applications, this method is not suitable for the commercial applications, as it requires high vacuum and high temperature leading to high cost.

\subsubsection{Hydrothermal Synthesis}

Hydrothermal method is defined as the crystallization of substance from organic or aqueous solution at high vapor pressure and temperature. Due to extreme conditions, this method is only suitable for those precursors which can withstand these harsh conditions. 2D layered nanostructured materials have been successfully synthesized by this approach. Single-layered $\mathrm{MoSe}_{2}$ and $\mathrm{MoS}_{2}$ nanostructures were obtained through chemical reaction of $\mathrm{Se} / \mathrm{S}$ with $\left(\mathrm{NH}_{4}\right)_{6} \mathrm{Mo}_{7} \mathrm{O}_{24} \cdot 4 \mathrm{H}_{2} \mathrm{O}$ (ammonium molybdite) in hydrazine monohydrate solution at a temperature of $150-180{ }^{\circ} \mathrm{C}$ for $48 \mathrm{~h}$ [117]. Furthermore, the single-step solvothermal reaction of hydrazine and $\left(\mathrm{NH}_{4}\right)_{2} \mathrm{MoS}_{4}$ on $\mathrm{GO}$, in $\mathrm{C}_{3} \mathrm{H}_{7} \mathrm{NO}$ ( $\mathrm{N}, \mathrm{N}$-dimethylformamide) solution has produced the 3 to 10 layered $\mathrm{MoS}_{2}$ flakes [118].

Besides the synthesis of the $\mathrm{MoS}_{2}$ mono and few layered 2D nanostructures, other 2D materials have also been fabricated by the hydrothermal approach. Transition metal (groups IV and V) chalcogenides 2D nanostructures have been synthesized from metal chloride in oleylamine [119]. In this process, chalcogen sources such as elemental selenium, sulfur, or compound $\mathrm{CS}_{2}$ are employed. When sulfur is used as the source, irregular shape and poor crystallinity of TMDC is obtained which is mainly due to the highly reactive radical formation. In contrast, better crystallinity can be achieved when using elemental Se as the source [119]. When $\mathrm{CS}_{2}$ is employed initially, $\mathrm{H}_{2} \mathrm{~S}$ is generated followed by its reaction with metal precursor, resulting in the formation of metal disulfide 2D layered crystals with 
variable lateral size in (001) plane. This growth is due to the low surface energy of this particular plane compared to (010) and (100) planes.

Besides the synthesis of TMDCs, this method has a potential to synthesize the 2D nanostructured sheets of hydroxides or metal oxides such as nanoribbons of $\mathrm{VO}_{2}$, which can be obtained by hydrothermal reduction in $\mathrm{V}_{2} \mathrm{O}_{5}$ in the presence of GOIt and is promising for applications in Li-ion batteries as a cathode [73]. Characterization techniques such as STM, X-ray photoelectron spectroscopy (XPS), and high-resolution transmission electron microscopy (HRTEM) have shown good composition control and crystal structure of $2 \mathrm{D}$ nanostructure materials synthesized by this approach.

\subsection{Properties and Applications of 2D Materials}

The layered materials in which one dimension is restricted to a single layer are called 2D materials. In 2D materials, the increment in excitation energy leads to an increase in the density of states [120], resulting in different properties with different sizes and shapes of the quantum confined 2D materials. 2D materials exhibit dissimilar properties than their bulk counterpart and demonstrate the shape- and sizedependent properties, which make them suitable for a variety of nanoapplications [121].

2D layered nanomaterials have electron confinement and the layers are in close contact by van der Waals force, which results in a minimization of the interlayer interaction. Large surface-to-volume ratio allows altering the properties through surface treatments such as chemical functionalization [122]. Moreover, 2D materials can also be synthesized as dispersed nanoflakes, which can retain their properties similar to the monolayers. In addition, these nanoflakes can be mixed with other materials to form nanocomposites, which have been widely used for energy applications [89, 123, 124]. Highly conductive nature coupled with large surface area, excellent chemical stability, and flexibility make 2D materials as suitable candidates for the applications of energy storage and conversion [16]. 2D materials can also be utilized in fuel cells due to their photocatalytic properties [125]. Moreover, anodes made from graphene have shown enhanced cyclic lithium storage capacity (specific capacity of $460 \mathrm{mAh} \mathrm{g}^{-1}$ ), which can be utilized in the flexible battery devices [126-128]. 2D materials have also shown promising properties for supercapacitors. Recently, supercapacitors made from multilayered reduced graphene oxide have demonstrated a high specific capacity of $394 \mu \mathrm{F} \mathrm{cm}{ }^{-2}$ [129].

Graphene has given rise to high charge carrier mobility, chiral properties of the mobile carriers, and high thermal conductivity [3]. Charge carriers in graphene can be well described by Dirac equation rather than the Schrödinger equation [130, 131]. It is well known that graphene is a gapless semiconductor. High symmetry of honeycomb lattice protects the zero gap of single-layer crystal. However, the band gap of bilayer graphene can reach up to $250 \mathrm{meV}$ by the application of a transverse electric field and the band gap is tunable by an electric field [132]. Besides electric field effect, strain can also alter the lowenergy band structure of layered 2D materials. For example, the band gap of a single- and bilayered $\mathrm{MoS}_{2}$ decreases linearly with strain at the rate of $\sim 45$ and $\sim 120 \mathrm{meV} / \%$, respectively [133, 134]. Furthermore, magnetic field also has a strong impact on electronic properties of $2 \mathrm{D}$ materials [43, 135, 136]. Charge carrier sign of 2D nanostructure materials can be modified by the application of electrical field allowing its utilization in high-mobility $\mathrm{p}-\mathrm{n}$ junction transistors and complementary metal oxide semiconductor (CMOS) technology. Defect-free high-quality crystal up to micron scale [137] is the main reason for achieving high charge carrier mobility due to scattering free movement of electrons [138]. Therefore, 2D materials are very popular in the design of nanoelectronic devices such as field-effect transistors (FET) due to their high charge carrier mobility, high on/off current ratios, and low power consumption. FET composed of pristine graphene exhibits very high electron mobility $\left(200,000 \mathrm{~cm}^{2} \mathrm{~V}^{-1} \mathrm{~s}^{-1}\right)$ [21] but lacks in on/off ratio. In contrast, some $2 \mathrm{D}$ materials such as $\mathrm{MoS}_{2}$ (TMDC) exhibit variable band gap (1.2 eV, indirect to $2.5 \mathrm{eV}$, direct from bulk to a single layer) [26-28, 139]. Indirect-to-direct transition of band gap from bulk to the single-layered 2D material is due to the upshift of the indirect band gap induced by strong quantum confinement effect in a single layer [26]. This intrinsic large band gap of such single-layered 2D material gives rise to $10^{8}$ on/off ratio and $\sim 150 \mathrm{~cm}^{2} \mathrm{~V}^{-1} \mathrm{~s}^{-1}$ electron mobility at $300 \mathrm{~K}$ for transistor application [30-33]. Furthermore, due to quantum limitations, channel thickness of $<5 \mathrm{~nm}$ cannot be achieved in Si-based devices, as it reduces the carrier mobility significantly due to scattering generated by surface roughness [140]. Thus, two-dimensionality of TMDCs gives the advantage of dangling bonds free, fully terminated surface [13]. In comparison with $\mathrm{Si}$ transistors, it demonstrates $10^{5}$ times less power consumption [30]. Similar to TMDC, 2D phosphorene has also shown promising results for transistor applications [141]. In addition, mechanical strains and electrical field both have significant influences on the band structure of 2D nanostructured materials, which makes them suitable candidates for sensor applications. High mobility results in highly sensitive conductivity (to electrostatic perturbation) of $2 \mathrm{D}$ materials due to the possible generation of carriers on the surface via photo (light)-effects [142]. This property makes these materials potential candidates for high-gain photodetector application such as optical communications, 
optoelectronic devices, and biomedical imaging. 2D materials can also be used as the nanogenerators [6] which convert the biomechanical energy, induced by the human body motion, into the electrical signal. This property makes them promising for biosensor and body implanted device applications [6, 143]. 2D materials can absorb a large range of the electromagnetic spectrum (infrared to ultraviolet) [144] allowing their utilization in high-performance photonics and optics [145]. Atomic layer graphene can be used for ultrafast photonics application due to its wavelength independent ultrafast saturable absorption [146]. 2D topological insulators (e.g., $\mathrm{Bi}_{2} \mathrm{Se}_{3}$ ) have the potential to be utilized for nonlinear optics applications at high-power regime (low absorption and high nonlinear phase) [147]. Few-layered 2D TMDC materials or $\mathrm{MoS}_{2}$ nanoplatelets possess nonlinear optical properties and can potentially be used in laser photonic devices [148]. Black phosphorus is also a promising nonlinear optical material. Thin films of black phosphorus can be used in developing ultrafast photonic devices [149]. 2D materials have very low absorbance value $(<10 \%)$ and high conductivity, making them suitable for flexible and transparent electronic applications such as liquid crystal devices and solar cells [150, 151].

Along with the extraordinary electrical and photonic properties, 2D materials also display excellent mechanical properties. The 2D material, such as graphene, is very flexible and has demonstrated 200 times higher breaking strength than steel [152]. Hence, it can be used to reinforce the polymers. Furthermore, its membrane can also be used in nonlinear electromechanical systems due to its extraordinary flexibility and ultrathin nature [153].

\section{Field-Effect Transistors}

\subsection{Introduction}

In general, the transistor is a device which controls the flow of the electrical charge carriers across a semiconductor material by which it is fabricated. Field-effect transistors (FETs) are the particular class of transistors in which semiconductor material is used as a channel. The current carrier density and conductivity of a semiconductor channel are controlled by an applied voltage resulted from the regulated current flow passing through it by a supply. Both ends of the channel connected to the input supply are denoted as the source and drain terminals. The terminal which is responsible for controlling the conductivity and current flow through the channel, upon the application of the potential, is known as a gate terminal, as shown in Fig. 2.

The basic principal of the FET is not new and was first described by J.E. Lilienfeld in a patent of 1925. The

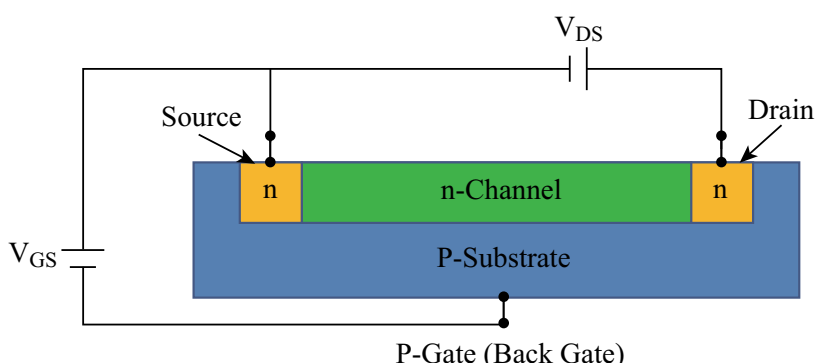

Fig. 2 Idealized N-channel junction FET

development of electronic devices was made possible after the theoretical description of the concept related to FET, given by Shockley in 1952 [154]. The general field-effect transistors can be categorized into two major classes, junction FET (JFET) and metal oxide semiconductor FET (MOSFET), also known as an insulated gate FET (IGFET), as shown in Fig. 3. In the era of 1970-1980, the invention of a MOSFET has led to the revolution of the electronic circuits and the development of the microprocessors, resulting in the powerful portable calculators and computers. FET has a variety of applications such as protection devices, amplifiers, switches, current limiters, oscillators, mixers, and voltage-controlled resistors.

\subsection{Potential of 2D Materials in FET}

Developments and advancements in materials have led to the exponential decrease in dimensions of the metal oxide semiconductor FET for over four past decades. This shrinking of size is the evidence of Moor's law prediction. These advancements not only scale down the size of silicon-based MOSFET but also enable it to perform even faster compared to its ancestors. However, this miniaturization and fast processing are possible at the cost of highpower consumption. This high-power requirement is due to the short-channel effect which arises in the MOSFET having a dimension less than $50 \mathrm{~nm}$ and makes the gate control weaken over the channel current. This results in disability of the gate to completely switch "off" the current through the channel [155]. This flow of unwanted leakage current, at the "switch off" state of MOSFET device, gives

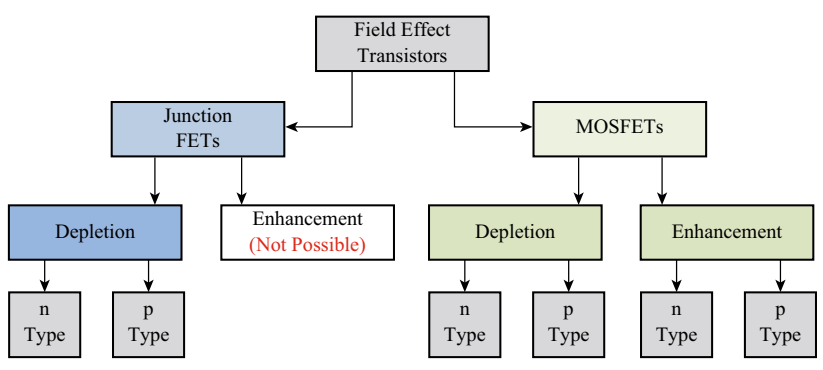

Fig. 3 FET tree 
rise to the high-power consumption and overheating problems. Further miniaturization of MOSFETs has been restricted due to the aforesaid challenges. To mitigate these complications and have better gate control to completely prevent the current flow through the device at "off" state, different approaches such as multiple gates [155] and high$\mathrm{k}$ dielectric gate layer [156] have been investigated. However, tunneling field-effect transistor (TFET), another type of FET, in which carrier transport is due to the interband tunneling, can also show high $\mathrm{I}_{\text {on }} / \mathrm{I}_{\text {off }}$ with a low supply voltage which is attributed to the subthreshold slope (SS) $<60 \mathrm{mV}$ per decade [157, 158]. The potential of TFETs was first realized through the theoretical simulations $[159,160]$. In addition, the research community is also looking for the alternate materials to complement or replace silicon technology. This brings the focus of researchers toward atomically thick mono or few layered 2D materials having unique characteristics appropriate for the future electronic devices, such as high mobility, transparency, and flexibility [161, 162]. The diverse properties of 2D materials, such as atomically thick body, robust nature, quantum confinement effect, high mobility, high switching efficiency, and tunable band gap, may result in the further scaling down of the device dimensions coupled with comparatively reduced short-channel effects [12, 21, 26, 30, 139]. In 2013, theoretical study, based on quantum transport simulations, shows promising results for reduction in channel length to $\sim 10 \mathrm{~nm}$ [163]. Recently, experimental results, of U-shape $\mathrm{MoS}_{2}$ FET with $10 \mathrm{~nm}$ channel length, have demonstrated excellent short-channel behaviors [164].

As described previously, 2D materials such as graphene and TMDC were fabricated from their bulk form due to the layered structure, which is held by a weak van der Waals force [1,20] although good ohmic contact, higher carrier mobility, and band gap $(\sim 1 \mathrm{eV})$ are the basic requirements for any material to be used in logic applications such as MOSFET, cost-effective large-scale synthesis of the material, and its compatibility with CMOS technology are also of great importance [165]. Moreover, 2D TMDCs also possess large relative effective mass for electrons $(\sim 0.5)$ and holes $(\sim 0.66)$ compared to Si $(\sim 0.29)$ [166] which results in reduced source-drain tunneling component in case of TFETs [167]. Researchers are still working hard to search new high-performance 2D materials or improve the current 2D materials for the requirements. In the past decade, significant progress has been achieved in the field of 2D layered materials for the state-of-the-art electronic nanodevices. However, there is still no 2D material meeting all the requirements for the high-performance 2D-based electronic device. For example, graphene shows very high mobility [21] but lacks in switching efficiency $(<10)$ at ambient temperature. On the other hand, $\mathrm{MoS}_{2}$ has high
$\mathrm{I}_{\mathrm{on}} / \mathrm{I}_{\text {off }}$ ratio $(\sim 108)$ [30] due to its relatively large band gap [26-28, 139] but has low electron mobility [30-32] compared to graphene. Many research works have shown that the number of the layers has a profound influence on the performance of electronic devices and the properties of layers can also be affected by the substrate or another 2D layered material coupled with them. Therefore, to form a heterostructure from two kinds of 2D materials, such as graphene and $\mathrm{MoS}_{2}$, may be employed to achieve and $\mathrm{I}_{\mathrm{on}} /$ $\mathrm{I}_{\text {off }}$ ratio simultaneously $[4,168]$. Along with the aforesaid essential properties, thermal conductivity and heat dissipation are also of vital importance for the realization and thermal management of the high-quality electronic device. The theoretical and experimental studies of the thermal behavior of 2D TMDCs, despite being the promising materials for FET applications, are still limited as compared to graphene and h-BN [169-173]. However, thermal properties of monolayer $\mathrm{MoS}_{2}$ (2D TMDC) differ from one-atom thin graphene layer due to its sandwich structure [174]. The thermal conductivity is mainly dominated by the phonons contribution rather than electrons [174]. Recently, theoretical investigations revealed the thermal conductivity of $\mathrm{MoS}_{2}$ is 1.35 [175], 6 [176] or $23.2 \mathrm{~W} \mathrm{~m}^{-1} \mathrm{~K}^{-1}$ [174] in different reports, which is lower in the magnitude than that of graphene. Moreover, experimental studies of $\mathrm{MoS}_{2}$ with a few layers have corroborated the thermal conductivity of 1.59 [177] and $52 \mathrm{~W} \mathrm{~m}^{-1} \mathrm{~K}^{-1}$ [178] at room temperature. Complete understanding of the thermal properties, of $\mathrm{MoS}_{2}$ (TMDCs), is crucial for the future state-of-the-art electronic device applications.

Along with the experimental research, semiconductorbased FET modeling and simulation serve as a bridge between manufacturers and designers $[179,180]$ and provide essential tools to explore the fundamental properties of 2D TMDCs for the device applications [181]. Various softwares (such as PHILIPAC, SLIC, and SPICE) are available to model and investigate the devices [182, 183]. These compact models for TMDC-based FETs are vital to study the device behaviors with computational efficiency and accuracy without loss of the physical insights [184]. Before the industrial-level production of the integrated circuits of FET based on TMDCs, the study of devices using the compact model is of prime importance. Compact model for analyzing these devices differs from Si-based devices because of the Fermi-Dirac statistics and density of states' effects on the capacitance [185-187]. Recently, many researchers have shown interest in developing the compact model for TMDC-based FETs. Each compact model has its own significance. For example, one model is based on capacitive network, which considers drift component but ignores trap effects [188]. Whereas, another model has more focus on the subthreshold region of the 
device [189]. One of the models is developed to simplify the current and surface potential calculations with the help of the Boltzmann statistics [190]. Later on, the model is developed, for double-gate FETs, based on Fermi-Dirac statistics which involves implicit equations but excludes the trap effects [187]. The research work in [191] demonstrates the compact model, which also considered the Fermi-Dirac statistics with drift-diffusion transport. These models were developed to explore the behavior of the TMDC channel-based FETs.

\subsection{Applications of 2D TMDC Materials in MOSFET}

Metal oxide semiconductor field-effect transistors (MOSFETs) currently are the main components of any logic devices, and each digital circuit is composed of many logic gates. Any material chosen to be used in the fabrication of logic devices needs to satisfy some basic requirements such as low conductance at off condition to minimize the standby power consumption, high on/off switching ratio from $10^{4}$ to $10^{7}$, and higher mobility of charge carrier for swift operation [192]. Large band gaps $(1.2-2.5 \mathrm{eV})$ of bulk, few- or single-layered $\mathrm{MoS}_{2}$ make it a suitable candidate to provide high switching ratio with low off-state power consumption $[27,28,30]$. Because of high switching ratio and large band gap, $\mathrm{MoS}_{2}$ being the potential candidate has been extensively studied for its application in the logic device. In this review, we will focus on $\mathrm{MoS}_{2}$-based FET as an example. Initially, low charge carrier mobility of $0.5-3 \mathrm{~cm}^{2} \mathrm{~V}^{-1} \mathrm{~s}^{-1}$, which is not sufficient for logic devices, was reported [29]. Subsequently, mechanically exfoliated nanopatches of $\mathrm{MoS}_{2}$ exhibited a high on/off ratio $>10^{5}$ and charge carrier mobility in tens of $\mathrm{cm}^{2} \mathrm{~V}^{-1} \mathrm{~s}^{-1}$ [193]. High switching on/off ratio of $10^{8}$, charge carrier mobility higher than $\sim 60 \mathrm{~cm}^{2} \mathrm{~V}^{-1} \mathrm{~s}^{-1}$, and subthreshold swing of $74 \mathrm{mV}$ per decade (increase in gate voltage required to change the drain current by one decade. Here decade represents the 10 times increment in drain current) were reported in top-gated $\mathrm{MoS}_{2}$-based FET at room temperature [30-32]. Subthreshold swing gives the qualitative and quantitative analysis of $\mathrm{I}_{\mathrm{on}} / \mathrm{I}_{\mathrm{off}}$. A lower value of subthreshold swing for FET will result in higher $\mathrm{I}_{\text {on }} / \mathrm{I}_{\text {off }}$ and better switching behavior. At room temperature, the ideal subthreshold value is $60 \mathrm{mV}$ per decade [13]. Similarly, monolayered $\mathrm{MoS}_{2}$ FET has also shown on/off ratio of $10^{8}$ and charge carrier mobility of $\sim 150 \mathrm{~cm}^{2} \mathrm{~V}^{-1} \mathrm{~s}^{-1}$ at $300 \mathrm{~K}$ once dielectric $\mathrm{HfO}_{2}$ was used as the gate layer [30-33, 194] (Fig. 4).

$\mathrm{MoS}_{2}$ showed low power consumption when high- $k$ dielectric gate layer in top-gated configuration was used. Besides the high performance in FET, photoresponsivity in
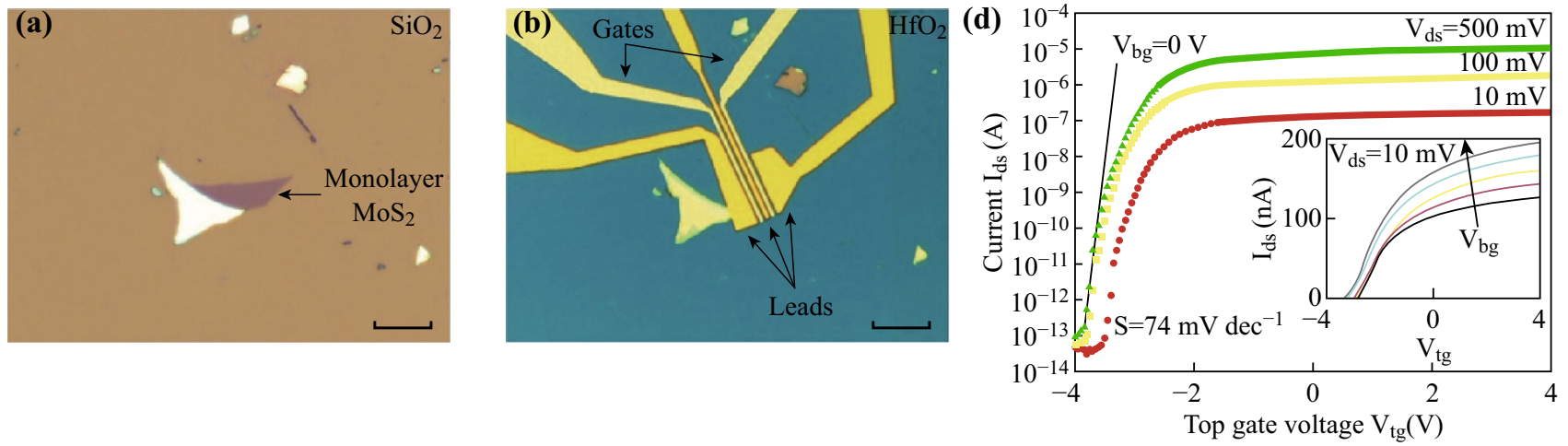

(c)

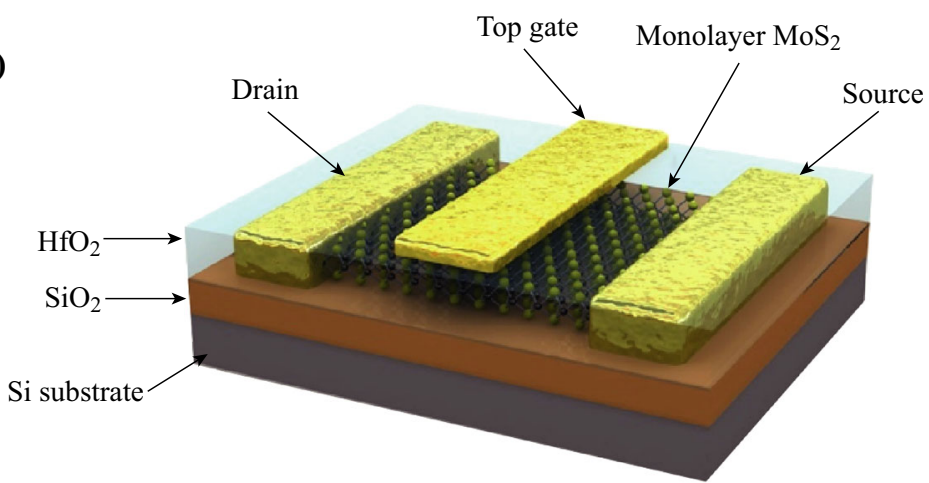

(e)

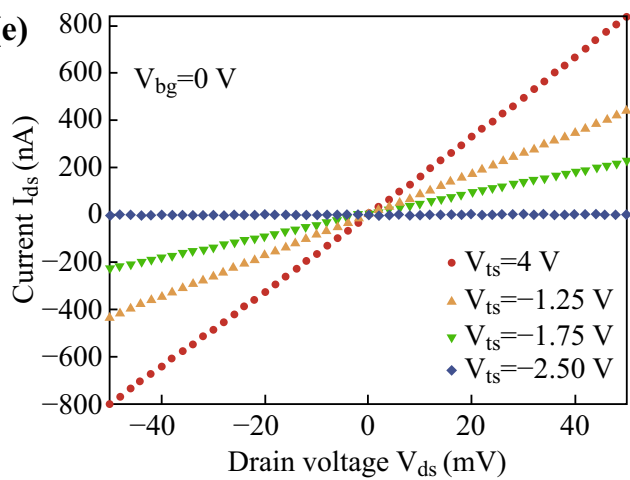

Fig. 4 a-c Preparation process of the single-layer $\mathrm{MoS}_{2}$ transistor. d and e the gate control of single-layer MoS 2 transistor. Reprinted with permission from Ref. [30], Copyright 2011, Nature Publishing Group 

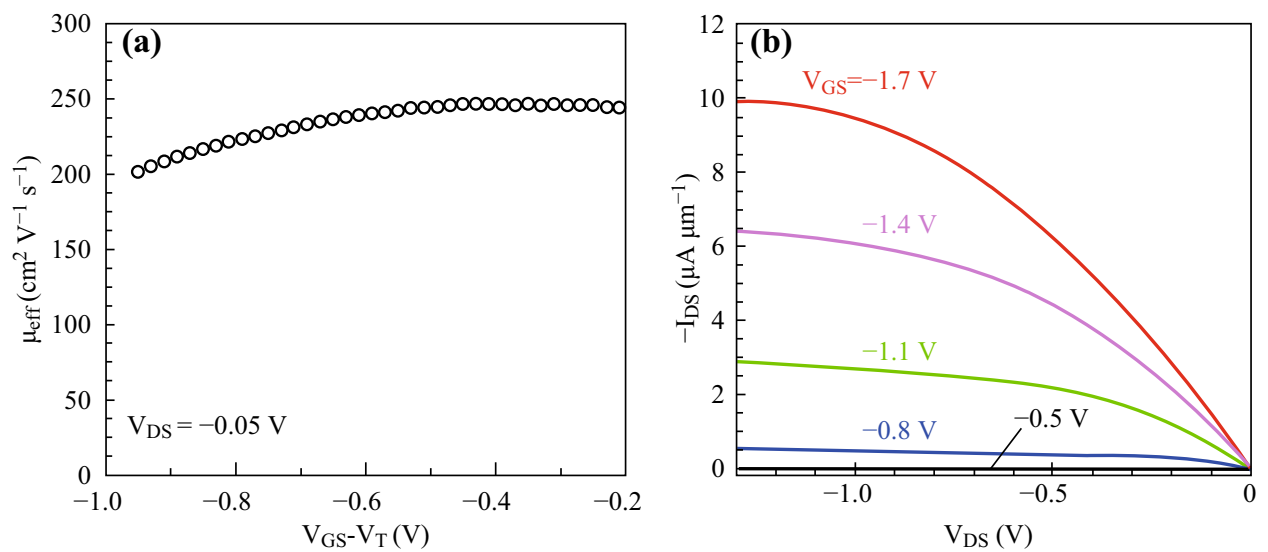

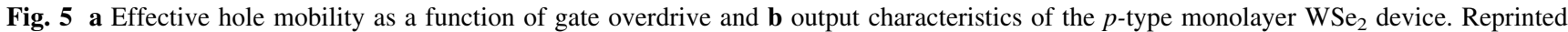
with permission from Ref. [168], Copyright 2012, American Chemical Society

the range of $100-680 \mathrm{~nm}$ and ultrasensitivity of $880 \mathrm{~A} \mathrm{~W}^{-1}$ at a wavelength of $561 \mathrm{~nm}$ was observed in monolayered $\mathrm{MoS}_{2}$ photodetectors [195]. The excellent optical properties of $\mathrm{MoS}_{2}$ make it a potential material for the applications in video recording, optoelectronics, light sensing, and biomedical imaging applications [195-197]. Besides $\mathrm{MoS}_{2}, \mathrm{WSe}_{2}$, a p-type TMDC, is also equipped with the set of properties required for the device applications, such as considerable bulk indirect and monolayered direct band gap (1.2-1.65 eV) [198, 199]. FET based on bulk $\mathrm{WSe}_{2}$ exhibited promising room-temperature hole (charge carrier) mobility of $\sim 500 \mathrm{~cm}^{2} \mathrm{~V}^{-1} \mathrm{~s}^{-1}$ [200]. However, it lacked in on/off current ratio $(>10)$. Bulk nature of $\mathrm{WSe}_{2}$ results into large "OFF" state current which is not desirable for the efficient FET applications. In contrast, mechanically exfoliated single-layered $\mathrm{WSe}_{2}-$ based p-type FET has shown the hole mobility of $\sim 250 \mathrm{~cm}^{2} \mathrm{~V}^{-1} \mathrm{~s}^{-1}$ with the enhanced on/off current ratio $\left(>10^{6}\right)$ and ideal subthreshold slope of $\sim 60 \mathrm{mV}$ per decade, as shown in Fig. 5 [199]. Moreover, p-type FET fabricated from CVD grown monolayer $\mathrm{WSe}_{2}$ has demonstrated the hole mobility of $\sim 90 \mathrm{~cm}^{2} \mathrm{~V}^{-1} \mathrm{~s}^{-1}$ with an appropriate on/off current ratio of $10^{5}$ [198, 201]. Similarly, $\mathrm{MoTe}_{2}$-based FETs show the ambipolar response. Both $\mathrm{n}$ - and p-type material can be synthesized by controlling the growth method [202]. Theoretical calculations have depicted the room-temperature mobility of $\mathrm{MoTe}_{2}$-based FET is of $\sim 200 \mathrm{~cm}^{2} \mathrm{~V}^{-1} \mathrm{~s}^{-1}$ [203]. However, the recent experimental works for three-layered $\mathrm{MoTe}_{2}$ device with $\mathrm{Ti}$ contacts have presented charge carrier mobility of $7 \times 10^{-2}$ and $2 \times 10^{-2} \mathrm{~cm}^{2} \mathrm{~V}^{-1} \mathrm{~s}^{-1}$ for holes and electrons, respectively [204, 205], whereas, in another work, a similar $\mathrm{MoTe}_{2}$ device with Au contacts has shown improved mobility of $16.5 \mathrm{~cm}^{2} \mathrm{~V}^{-1} \mathrm{~s}^{-1}$ with on/off current of $10^{7}$ [204].
Regarding the current research of TMDCs on FET, charge carrier mobility is the main focus. The impurities presented in mono or few layered $\mathrm{MoS}_{2}$ material lead to screening effect, which influences the dielectric environment on charge carrier mobility. The large dielectric constant of the gate layer will increase the capacitive coupling between top gate and back gate which in return stimulates the charge carrier mobility by $10-50$ times [194]. If only silicon/silicon dioxide is used as the substrate without high-k dielectric gate layer in mono or few layered $\mathrm{MoS}_{2}$-based FET, charge carrier mobility of about $10 \mathrm{~cm}^{2} \mathrm{~V}^{-1} \mathrm{~s}^{-1}$ can be obtained at room temperature. Hence, the low mobility is due to the poor interface between $\mathrm{Si} / \mathrm{SiO}_{2}$ and $\mathrm{MoS}_{2}$ [206, 207]. This interface problem including surface defects, the concentration of charged impurities, local charge distribution, and trapped charges in the substrate results in coulomb scattering and low charge carrier mobility. The use of high-k dielectric materials, such as hafnium dioxide $\left(\mathrm{HfO}_{2}\right)$ [30-32] or alumina $\left(\mathrm{Al}_{2} \mathrm{O}_{3}\right)$ [208] as top gate, has increased the charge carrier mobility to $\sim 150$ and $500 \mathrm{~cm}^{2} \mathrm{~V}^{-1} \mathrm{~s}^{-1}$, for monolayer and bulk $\mathrm{MoS}_{2}$, respectively, due to the screening effect. Besides the influences of high- $k$ dielectric gate layer and channel material $\left(\mathrm{MoS}_{2}\right)$ characteristics, charge carrier mobility has been underestimated due to the presence of Schottky barriers between metallic contacts and $\mathrm{MoS}_{2}$ single or few layers [194, 209]. The reduction in resistance between the contacts and thinning of Schottky barrier results in an enhancement of charge carrier mobility from 100 to $220 \mathrm{~cm}^{2} \mathrm{~V}^{-1} \mathrm{~s}^{-1}$ [210]. Theoretical calculations based on density function theory suggested that the carrier mobility of $\mathrm{MoS}_{2}$ at room temperature could reach $400 \mathrm{~cm}^{2} \mathrm{~V}^{-1} \mathrm{~s}^{-1}$ [34]. These results suggest that $\mathrm{MoS}_{2}$ is a promising material for future electronics. 


\section{Current Challenges in 2D Materials Device Application}

Experimentally demonstrated charge carrier mobility of the mono or few layered $\mathrm{MoS}_{2}$ is much lower than that of theoretical prediction of $410 \mathrm{~cm}^{2} \mathrm{~V}^{-1} \mathrm{~s}^{-1}$ [34]. 2D materials possess large surface area making charge carrier mobility more sensitive to the external (e.g., trapped charged impurities, interface quality, and adsorbates from ambient air) and internal (lattice defects) factors [206, 207]. An experimental result of charge carrier mobility depends on the measurement condition/method and sample quality. Other factors including channel thickness, measuring temperature, annealing, Schottky barrier, dielectric environment, and carrier density (n) play an important role in achieving high charge carrier mobility devices.

The influence of channel thickness on carrier mobility was ascertained when mechanically exfoliated $\mathrm{MoS}_{2}$ and $\mathrm{NbSe}_{2}$ demonstrated $0.5-3.0 \mathrm{~cm}^{2} \mathrm{~V}^{-1} \mathrm{~s}^{-1}$ charge carrier mobility, which is lower than their bulk counterparts [29]. Lee et al. [211] also showed the reduction in carrier mobility with the decrease in thickness placed on different substrates $\left(\mathrm{SiO}_{2}\right.$ and h-BN). One- to 5-layered $\mathrm{MoS}_{2}$ displayed charge carrier mobility varying from 10 to $50 \mathrm{~cm}^{2} \mathrm{~V}^{-1} \mathrm{~s}^{-1}$ [212]. The possible reasons for the thickness dependence are coulomb scattering and the Schottky barrier between channel and electrode contacts [213]. Moreover, in recent work, Hall mobility of $\sim 1000$ and $\sim 34,000 \mathrm{~cm}^{2} \mathrm{~V}^{-1} \mathrm{~s}^{-1}$ is reported for single- and six (6)-layered encapsulated $\mathrm{MoS}_{2}$ devices, respectively, at a temperature below $5 \mathrm{~K}$ [214]. Just like the channel thickness, measuring or operating temperature has a significant effect on carrier mobility. High temperature $(>100 \mathrm{~K})$ enhances the lattice phonon scattering which tends to have an adverse effect on carrier mobility of 2D TMDCs. Consequently, the theoretically predicted charge carrier mobility of $410 \mathrm{~cm}^{2} \mathrm{~V}^{-1} \mathrm{~s}^{-1}$ at room temperature [34, 215], limited by phonon scattering, has never been achieved experimentally for single-layered $\mathrm{MoS}_{2}$ [216]. Similar behavior has also been reported for $\mathrm{MoSe}_{2}$ with a few layers [217]. At low temperatures, high field-effect mobility $\left(\sim 1000\right.$ and $\left.\sim 34,000 \mathrm{~cm}^{2} \mathrm{~V}^{-1} \mathrm{~s}^{-1}\right)$ of singleand six-layered $\mathrm{MoS}_{2}$ has been reported [214, 216]. Moreover, charge carrier density $(n)$ also plays a vital role in electronic properties of 2D TMDCs materials. $\mathrm{MoS}_{2}$ has shown electronic phase transition (metal to insulator) with an increase in charge carrier density [218, 219], whereas medium range charge carrier density is essential for the transistor applications. In this range, an increase in charge density may have two outcomes. First, high charge density is favorable for increasing carrier mobility by suppressing the interfacial impurity potential. Second, high carrier density tends to increase the carrier energy which may result in reducing carrier mobility [212].

Interfacial Schottky barrier, between the channel semiconductor and metal electrodes, reduces the charge carrier mobility by inducing resistance in carrier transfer. This unwanted effect can be minimized by employing four-terminal measurement method [220]. Formation of the Schottky barrier is due to the difference in energy levels between the semiconductor and electrode materials. Schottky barrier height is directly proportional to the energy-level difference of two coupled materials [221]. Barrier height can be reduced by making contact between $n$-type semiconductor and low work function metals or $p$ type semiconductor and high work function metal. Moreover, the Schottky barrier is tunable by varying the FET gate bias. Initially, this variation was considered to be the switching mechanism of FETs [222]. Later, the dependence of barrier height on channel thickness was reported. In thicker flakes, the barrier height is much smaller than that of monolayered 2D TMDCs materials [223]. It is due to the influence of band gap of semiconductor channel on the Schottky barrier. Thinner ( $<5$ layers) $\mathrm{MoS}_{2}$ possesses larger band gap which results in higher barrier height and contact resistivity than bulk $\mathrm{MoS}_{2}$ [220]. Barrier height between $\mathrm{Au}$ and $\mathrm{MoS}_{2}$ varies from 0.3 to $0.6 \mathrm{eV}$ with a decrease in the number of $\mathrm{MoS}_{2}$ layers from 5 to 1 [220]. Hence, charge carrier mobility can be improved by optimizing the metal/semiconductor contact. Furthermore, minimum electrode length needs to be identified in order to obtain better charge transferring efficiency [220]. Channel length (in appropriately annealed samples), however, does not affect the $\mathrm{MoS}_{2}$ device performance [220]. In addition, some studies have shown that the presence of ultrathin $\mathrm{TiO}_{2}$ [224] or $\mathrm{MgO}$ [225], between $\mathrm{MoS}_{2}$ and ferromagnetic metal, reduces interfacial Schottky barrier height.

Scattering in semiconductor channel is one of the reasons for the reduced charge carrier mobility. The scattering may be induced by lattice phonons due to the high- $\mathrm{k}$ dielectric environment, charged impurities, and interfacial phonons. At room temperature, bulk $\mathrm{MoS}_{2}$ has exhibited the electron mobility ranging from $\sim 150$ to $500 \mathrm{~cm}^{2}$ $\mathrm{V}^{-1} \mathrm{~s}^{-1}[30-33,208]$. However, if the monolayers of $\mathrm{MoS}_{2}$ fabricated by mechanical exfoliated are transferred on $\mathrm{SiO}_{2}$ to make a device, the carrier mobility then drops down to $0.1-10 \mathrm{~cm}^{2} \mathrm{~V}^{-1} \mathrm{~s}^{-1}$ [29]. High charged impurity density $(N)$ gives rise to coulomb scattering which can be screened with the use of high- $k$ dielectric material, leading to the increase in electron mobility at low temperatures [219]. In FETs made of 2D TMDC materials, charged impurities (coulomb) are present at the interface between dielectric and 2D channel. These charged impurities have a 
scattering potential which induces the scattering in $2 \mathrm{D}$ FETs [226-228]. The source of charged impurities includes chemical residues, adsorbates introduced during device fabrication, and contaminated surface, which results in reduced charge carrier mobility of the 2D TMDC-based FETs. Theoretical calculations and experimental results have demonstrated a high rate of coulomb scattering in the thinner channel with $\mathrm{HfO}_{2}$ or $\mathrm{SiO}_{2}$ as the gate layer [212], indicating that the top/bottom surface scattering is associated with channel thickness. As the thickness decreases, interaction distance between charge carriers and charged impurities shrinks due to the electrostatic equilibrium. This shrink in interaction distance results in high scattering potential and lower charge carrier mobility [212].

Charge carriers can be scattered by lattice phonons through potential deformation. Adjacent atoms move in the direction of acoustic phonons but opposite to the optical phonons. Phonon scattering is proportional to temperature and increases with the increase in temperature. Based on theoretical calculations (by the first principals calculations) of acoustic/polar phonon scattering and screening for single-layered $\mathrm{MoS}_{2}$, charge carrier mobility of $\sim 410 \mathrm{~cm}^{2} \mathrm{~V}^{-1} \mathrm{~s}^{-1}$ has been reported $[215,229,230]$. However, these calculations did not cater for the effects of dielectric mismatch and free-carrier screening. Phonon scattering becomes dominant due to the presence of high- $k$ dielectric material and leads to a drastic decrease in electron mobility at room temperature (300 K) [230]. This phenomenon tends to increase as the thickness of the semiconductor layer is decreased from bulk to monolayer. In TMDC materials like $\mathrm{MoS}_{2}$, polar nature of the chemical bonds gives rise to the dipole moments between anions and cations. Perturbation of dipole moment by polar phonons creates an electric field which is coupled with charge carrier, resulting in low charge carrier mobility. This phenomenon is called Frohlich interaction or polar optical phonon scattering [229]. Phonons can be excited by the charge carriers if the dielectric layer in FETs supports polar vibrational modes. These phonons are known as surface optical phonons or remote interface phonons. At room temperature, scattering due to remote interface phonons is more dominant in the high-k dielectric environment compared to low $k$ dielectric gate layer [231, 232].

Besides coulomb and phonon scattering, structural defects also play a vital role in the degradation of charge carrier mobility. Structural defects include dislocation, vacancies, grain boundaries, impurities, and precipitation. In a low-quality sample, anion vacancies can act as a strong scattering source. Studies have shown that CVD grown or mechanically exfoliated $\mathrm{MoS}_{2}$ possesses high percentages $(0.4 \%)$ of sulfur vacancy which affects the charge carrier mobility [233]. Vacancy repairing of CVD grown single- layer $\mathrm{MoS}_{2}$ by annealing can improve the charge carrier mobility up to $45 \mathrm{~cm}^{2} \mathrm{~V}^{-1} \mathrm{~s}^{-1}$ [234]. Vacancy scattering is independent of carrier density and weakly depends on the temperature and channel thickness as these parameters do not have any direct relation with the defect densities [235]. Besides vacancy defects, the presence of tilt grain boundaries may also degrade the charge carrier transport efficiency [236].

Electron or charge carrier density $(n)$ is also one of the factors affecting the charge carrier mobility. Although electron mobility tends to increase with the increase in electron density, the effect is prominent after the effective screening of phonon scattering. As the dielectric value of $k$ increases, electron mobility drastically decreases due to phonon scattering. Electrons presented in atomically thick 2D mono or few layer nanosheets excite the phonons in the nearby dielectric material (which supports polar vibrational modes) at room temperature. Figure 6a [230] demonstrates the effects of dielectric environment on charge carrier mobility at two different temperatures of 100 and $300 \mathrm{~K}$. In both cases, electron density and charged impurity density are considered equal to $1013 \mathrm{~cm}^{-2}$. The solid line represents the combined effects of coulomb and phonon scattering on mobility, whereas the broken line shows the electron mobility while neglecting the effects of phonon scattering, which indicates a trend to achieve large mobility at high-k dielectric environment $\left(\varepsilon_{\mathrm{e}}\right)$. In this case, mobility is entirely dependent upon the charged impurity density.

At low temperature, when the phonon scattering is inactive and negligible, electron mobility can be improved by decreasing the charged impurity density. If the charged impurities density is low, low $k$ dielectric is required for coulomb screening, which will result in less phonon scattering at room temperature [230]. Figure 6b [230] shows the dielectric constant required against the critical value of charged impurity density $N_{\mathrm{cr}}$ at room temperature. If $N>N_{\text {cr }}$, then the electron mobility highly depends upon $N$ rather than phonon scattering and vice versa. Figure $6 \mathrm{c}$ [230] represents the charge carrier mobility of a monolayer $\mathrm{MoS}_{2}$ (phonon limited) at room temperature under different dielectric environment ranging from free suspension in the air to high- $k$ dielectric $\mathrm{HfO}_{2}$. It can be inferred from the plot that the phonon determined mobility decreases with the increase in dielectric constant of the gate layer. Figure $6 \mathrm{~d}[230]$ demonstrates the combined room-temperature electron mobility in monolayer $\mathrm{MoS}_{2}$ nanosheet against variable charged impurity densities under various dielectric environments considering all kinds of scattering mechanisms. The carrier density is fixed to be $1013 \mathrm{~cm}^{-2}$. From Fig. 6d, one can figure out that the electron mobility is weakly dependent on the dielectric environment at high impurity density $\left(1013 \mathrm{~cm}^{-2}\right)$, as shown in the dashed box in the bottom right corner. 

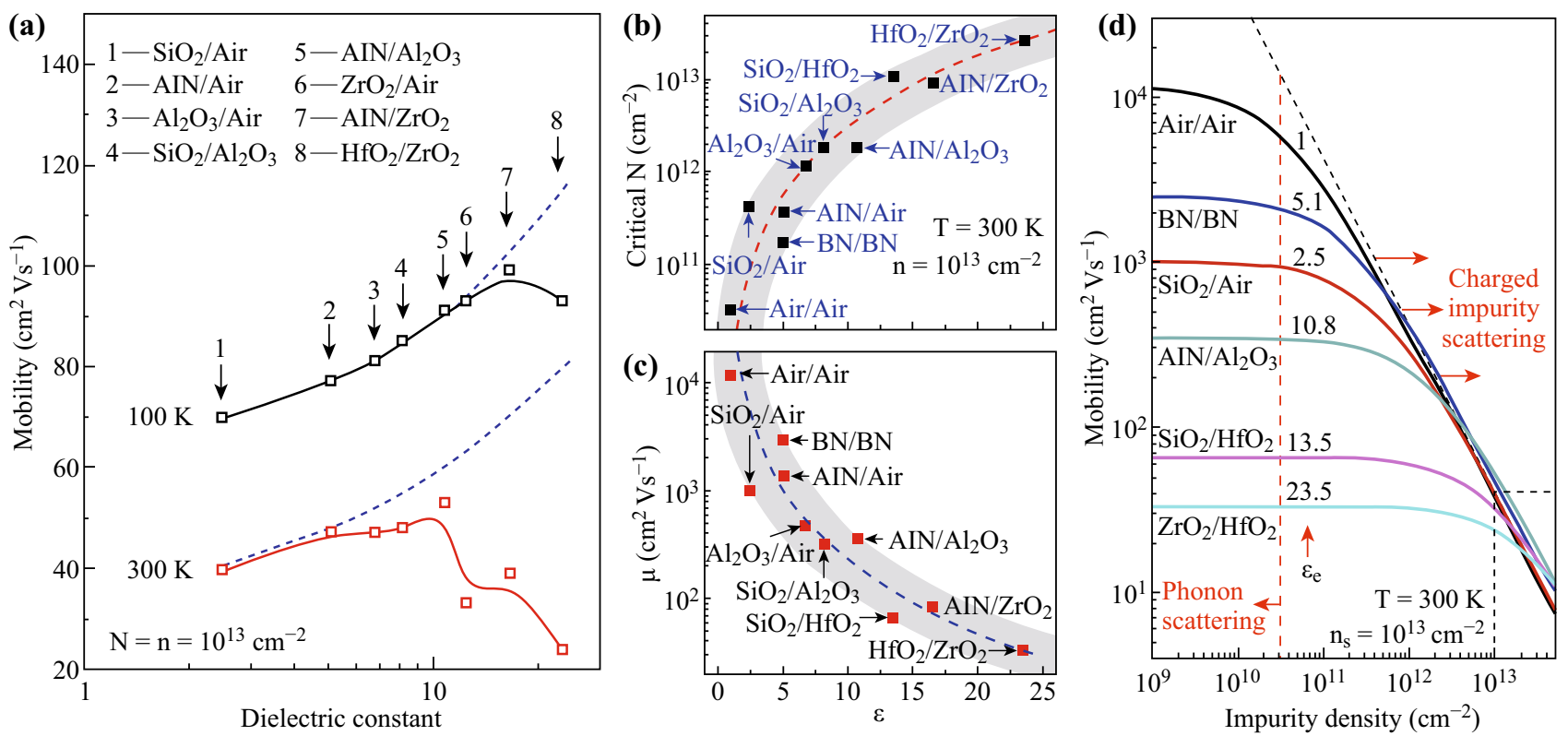

Fig. 6 a Electron mobility as a function of dielectric constant. Dashed lines show the mobility without considering the SO phonons. b The critical impurity densities Ncr and $\mathbf{c}$ Room-temperature electron mobility in monolayer $\mathrm{MoS}_{2}$ surrounded by different dielectrics. d The roomtemperature net electron mobility in monolayer $\mathrm{MoS}_{2}$ considering all kinds of scattering mechanisms as a function of impurity density $(N)$. Reproduced from Ref. [230]

\section{Future Prospective and Conclusion}

Aforementioned challenges, regarding the utilization of $2 \mathrm{D}$ nanomaterials in device applications in connection with the charge carrier mobility, drive us not only to produce the high-quality (defect- and impurity-free) single-crystal mono or 2D nanosheets with a few layers but also to think about searching new materials. This path leads to two methodologies pertaining to the improvement of charge carrier mobility by the utilization of existing materials via special design, such as heterostructure technique or experimental study of some new materials, which is possible to have high carrier mobility.

For the first methodology, efforts need to be made to produce the high-quality and defect-free 2D nanostructure which contains a minimum level of charged impurity density. Mechanical exfoliation can produce high-quality TMDCs. However, the difficulty for large-scale synthesis with uniformity and reproducibility of samples are the main limitations of this process. CVD is a method which has the potential of producing the large-scale high-quality 2D TMDCs or graphene $[237,238]$. In order to obtain the impurity-free high-quality 2D materials, the ultraclean substrate (flushing of the substrate with acetone and isopropanol and subsequently vacuum annealing at high temperature) and highly pure precursors need to be used. In addition, the synthesis parameters such as pressure, temperature, and growth time are needed to be optimized, which are all important to achieve large-scale, high-quality single-crystal 2D materials [237-239]. Proper procedures to avoid contamination during 2D material synthesis are also of importance. For example, the purging of $\mathrm{Ar}$ gas with a very high purity is required to eliminate any chance of impurity in the samples [240]. Thickness and number of layers of 2D material can be controlled by controlling the deposition parameters, such as heating temperature, deposition time, and chamber pressure [238, 241]. Furthermore, very recent developments have shown that adding a small amount of $\mathrm{O}_{2}$ in the Ar carrier gas can also suppress the nucleation and promote the synthesis of $\mathrm{MoS}_{2}$ with a large area and high quality [239]. In addition, device fabrication and encapsulation inside Ar-filled glove box will also help avoid device degradation, possibly due to the presence of chemical species (e.g., $\mathrm{O}_{2}$ and $\mathrm{H}_{2} \mathrm{O}$ ) in ambient condition [242].

From the above discussions, charge carrier mobility in 2D materials is primarily dependent on the concentration of the charged impurities and then on the phonon scattering due to the dielectric environment at high temperatures. CVD method has the potential to generate high-quality large-scale single-crystal 2D nanosheets. $\mathrm{MoS}_{2}$, graphene, $\mathrm{WS}_{2}$, and $\mathrm{MoTe}_{2}$ high-quality monolayers have been extensively reported using CVD techniques. Recently, it has been observed that assembling different 2D materials into heterostructure may lead to the tuning of electronic properties [243, 244]. A band gap of $0.1 \mathrm{eV}$ of $\mathrm{MoS}_{2}$ was achieved after a heterostructure composed of $\mathrm{MoS}_{2}$, and graphene was formed [243]. Furthermore, heterostructure- 
(a) $\odot \mathrm{Mo}$

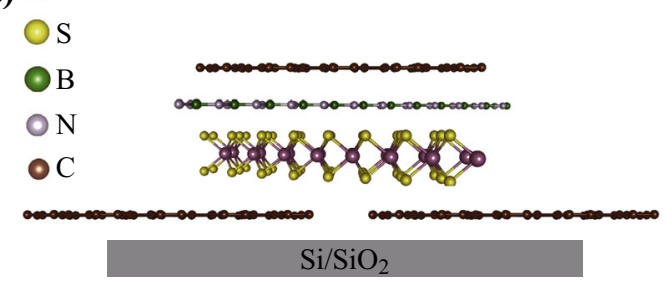

(b)

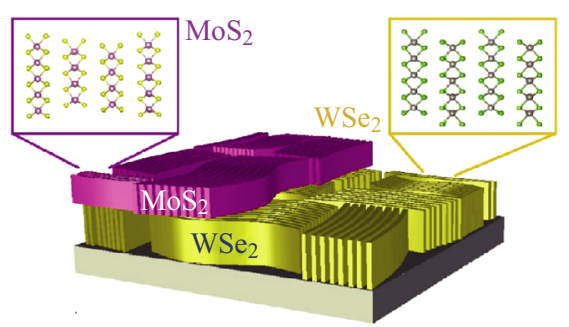

Fig. 7 Heterostructure-based a FET composed of $\mathrm{MoS}_{2}$ (channel), h-BN (top-gate dielectric), and graphene (source, drain, and top-gate electrodes). Redrawn from Ref. [245] and b p-n junction fabricated from vertical stacked $\mathrm{MoS}_{2}$ ( $n$ type) and $\mathrm{WSe}_{2}$ ( $p$ type). Reprinted with permission from Ref. [247]. Copyright (2015) American Chemical Society

based FET composed of $\mathrm{MoS}_{2}$ (channel), h-BN (top-gate dielectric), and graphene (source, drain and top-gate electrodes) has displayed charge carrier mobility of $\sim 33 \mathrm{~cm}^{2} \mathrm{~V}^{-1} \mathrm{~s}^{-1}$ and on/off current ratio of $>10^{6}$ [214, 245] (Fig. 7a). Besides $\mathrm{MoS}_{2}$, TMDCs p-n junction (heterostructure) also demonstrates promising results. Heterostructure of TMDCs, n-MoSe $2 / \mathrm{p}-\mathrm{WSe}_{2}$, has exhibited the clear rectification with an ideality factor of $\sim 2$ at 290 K [246]. Similarly, p-n junction of p-WSe $2 / n-\mathrm{MoS}_{2}$ (Fig. 7b) has also shown the promising gate tunable rectifying electrical characteristics [247-250]. The average conductance slope of $75 \mathrm{mV}$ per decade at room temperature [250] and ideality factor of 1.2 were observed [251]. However, in recent work, the $\mathrm{p}-\mathrm{n}$ heterojunction formed by $p$-type single-walled carbon nanotubes and $n$-type monolayer $\mathrm{MoS}_{2}$ has also revealed the charge transport behavior with a forward/reverse current ratio of larger than $10^{4}$ [252]. In addition, heterostructure made from InAs ( $n$ type)/ $/ \mathrm{WSe}_{2}$ ( $p$ type) has displayed the even better rectification with a forward/reverse current ratio larger than $10^{6}$ and an ideality factor of $\sim 1.1$ [253].

Secondly, to search and fabricate new materials may be another possible methodology to achieve high carrier mobility 2D materials. Recently, 2D material, InSe (metal chalcogenide)-based heterostructure encapsulated device has been reported, which demonstrates charge carrier mobility of $\sim 10,000$ and $\sim 1000 \mathrm{~cm}^{2} \mathrm{~V}^{-1} \mathrm{~s}^{-1}$ at $\sim 50$ and $300 \mathrm{~K}$, respectively [242]. Theoretical calculations have revealed that some new promising single-layer $2 \mathrm{D}$ TMDC materials may achieve higher mobility than that of

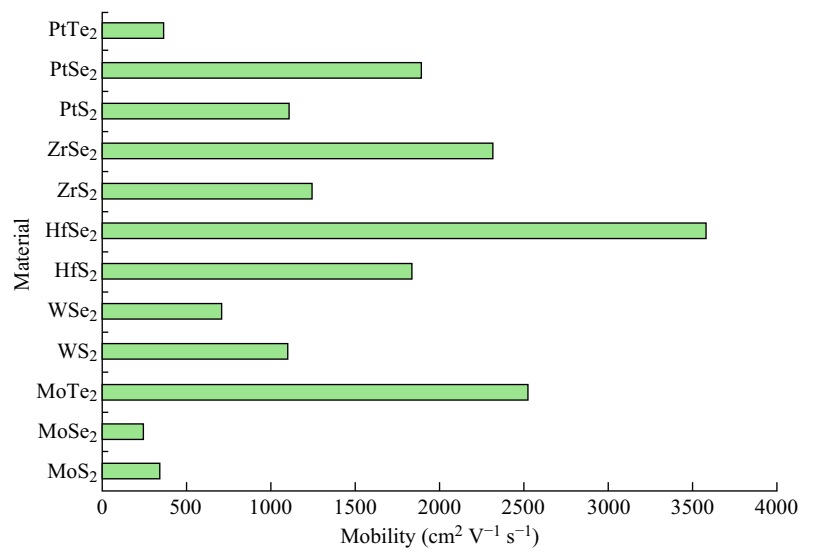

Fig. 8 Theoretical charge carrier mobility of monolayer 2D TMDC materials. Redrawn from Ref. [254]

$\mathrm{MoS}_{2}$ [254] as shown in Fig. 8. Certainly, the synthesis of these high-quality $2 \mathrm{D}$ materials is a challenge.

Acknowledgements This work is funded by Australian Research Council discovery project DP140103041 and Future Fellowship FT160100205.

Open Access This article is distributed under the terms of the Creative Commons Attribution 4.0 International License (http://crea tivecommons.org/licenses/by/4.0/), which permits unrestricted use, distribution, and reproduction in any medium, provided you give appropriate credit to the original author(s) and the source, provide a link to the Creative Commons license, and indicate if changes were made.

\section{References}

1. Q.H. Wang, K. Kalantar-Zadeh, A. Kis, J.N. Coleman, M.S. Strano, Electronics and optoelectronics of two-dimensional transition metal dichalcogenides. Nat. Nanotechnol. 7(11), 699-712 (2012). doi:10.1038/nnano.2012.193

2. J.A. Wilson, F.J. Di Salvo, S. Mahajan, Charge-density waves in metallic, layered, transition-metal dichalcogenides. Phys. Rev. Lett. 32(16), 882-885 (1974). doi:10.1103/PhysRevLett.32.882

3. A. Gupta, T. Sakthivel, S. Seal, Recent development in 2D materials beyond graphene. Prog. Mater Sci. 73, 44-126 (2015). doi:10.1016/j.pmatsci.2015.02.002

4. X. Li, H. Zhu, Two-dimensional $\mathrm{MoS}_{2}$ : properties, preparation, and applications. J. Materiomics 1(1), 33-44 (2015). doi:10. 1016/j.jmat.2015.03.003

5. G.R. Bhimanapati, Z. Lin, V. Meunier, Y. Jung, J. Cha et al., Recent advances in two-dimensional materials beyond graphene. ACS Nano 9(12), 11509-11539 (2015). doi:10.1021/ acsnano. 5 b05556

6. S.J. Kim, K. Choi, B. Lee, Y. Kim, B.H. Hong, Materials for flexible, stretchable electronics: graphene and 2D material. Annu. Rev. Mater. Res. 45(1), 63-84 (2015). doi:10.1146/ annurev-matsci-070214-020901

7. S. Das, J.A. Robinson, M. Dubey, H. Terrones, M. Terrones, Beyond Graphene: progress in novel two-dimensional materials and van der Waals solids. Annu. Rev. Mater. Res. 45(1), 1-27 (2015). doi:10.1146/annurev-matsci-070214-021034 
8. X. Tong, E. Ashalley, F. Lin, H. Li, Z.M. Wang, Advances in $\mathrm{MoS}_{2}$-based field effect transistors (FETs). Nano-Micro Lett. 7(3), 203-218 (2015). doi:10.1007/s40820-015-0034-8

9. S.Z. Butler, S.M. Hollen, L. Cao, Y. Cui, J.A. Gupta et al., Progress, challenges, and opportunities in two-dimensional materials beyond graphene. ACS Nano 7(4), 2898-2926 (2013). doi: $10.1021 / \mathrm{nn} 400280 \mathrm{c}$

10. D. Jariwala, V.K. Sangwan, L.J. Lauhon, T.J. Marks, M.C. Hersam, Emerging device applications for semiconducting twodimensional transition metal dichalcogenides. ACS Nano 8(2), 1102-1120 (2014). doi:10.1021/nn500064s

11. F. Xia, H. Wang, D. Xiao, M. Dubey, A. Ramasubramaniam, Two-dimensional material nanophotonics. Nat. Photon. 8(12), 899-907 (2014). doi:10.1038/nphoton.2014.271

12. X.R. Wang, Y. Shi, R. Zhang, Field-effect transistors based on two-dimensional materials for logic applications. Chin. Phys. B 22(9), 098505 (2013). doi:10.1088/1674-1056/22/9/098505

13. G. Fiori, F. Bonaccorso, G. Iannaccone, T. Palacios, D. Neumaier, A. Seabaugh, S.K. Banerjee, L. Colombo, Electronics based on two-dimensional materials. Nat. Nanotechnol. 9(10), 768-779 (2014). doi:10.1038/nnano.2014.207

14. C. Nitin, R.I. Muhammad, K. Narae, T. Laurene, J. Yeonwoong, I.K. Saiful, Two-dimensional lateral heterojunction through bandgap engineering of $\mathrm{MoS}_{2}$ via oxygen plasma. J. Phys.: Condens. Matter. 28(36), 364002 (2016). doi:10.1088/09538984/28/36/364002

15. H.-Y. Chang, M.N. Yogeesh, R. Ghosh, A. Rai, A. Sanne, S. Yang, N. Lu, S.K. Banerjee, D. Akinwande, Large-area monolayer $\mathrm{MoS}_{2}$ for flexible low-power RF nanoelectronics in the GHz regime. Adv. Mater. 28(9), 1818-1823 (2016). doi:10. 1002/adma.201504309

16. F. Bonaccorso, L. Colombo, G. Yu, M. Stoller, V. Tozzini, A.C. Ferrari, R.S. Ruoff, V. Pellegrini, Graphene, related two-dimensional crystals, and hybrid systems for energy conversion and storage. Science 347(6217), 1246501 (2015). doi:10.1126/ Science 1246501

17. N. Choudhary, M.D. Patel, J. Park, B. Sirota, W. Choi, Synthesis of large scale $\mathrm{MoS}_{2}$ for electronics and energy applications. J. Mater. Res. 31(7), 824-831 (2016). doi:10.1557/jmr.2016.100

18. V.J. Babu, S. Vempati, T. Uyar, S. Ramakrishna, Review of one-dimensional and two-dimensional nanostructured materials for hydrogen generation. Phys. Chem. Chem. Phys. 17(5), 2960-2986 (2015). doi:10.1039/C4CP04245J

19. S.S. Varghese, S.H. Varghese, S. Swaminathan, K.K. Singh, V. Mittal, Two-dimensional materials for sensing: graphene and beyond. Electronics 4(3), 651-687 (2015). doi:10.3390/ electronics 4030651

20. M. Xu, T. Liang, M. Shi, H. Chen, Graphene-like two-dimensional materials. Chem. Rev. 113(5), 3766-3798 (2013). doi:10. $1021 / \mathrm{cr} 300263 \mathrm{a}$

21. K.I. Bolotin, K.J. Sikes, Z. Jiang, M. Klima, G. Fudenberg, J. Hone, P. Kim, H.L. Stormer, Ultrahigh electron mobility in suspended graphene. Solid State Commun. 146(9), 351-355 (2008). doi:10.1016/j.ssc.2008.02.024

22. X. Wang, Y. Ouyang, X. Li, H. Wang, J. Guo, H. Dai, Roomtemperature all-semiconducting sub-10-nm graphene nanoribbon field-effect transistors. Phys. Rev. Lett. 100(20), 206803 (2008). doi:10.1103/PhysRevLett.100.206803

23. Y. Ding, Y. Wang, J. Ni, L. Shi, S. Shi, W. Tang, First principles study of structural, vibrational and electronic properties of graphene-like $\mathrm{MX}_{2}(\mathrm{M}=\mathrm{Mo}, \mathrm{Nb}, \mathrm{W}, \mathrm{Ta} ; \mathrm{X}=\mathrm{S}, \mathrm{Se}, \mathrm{Te})$ monolayers. Phys. B Condens. Matter. 406(11), 2254-2260 (2011). doi:10.1016/j.physb.2011.03.044

24. C. Ataca, H. Şahin, S. Ciraci, Stable, single-layer $\mathrm{MX}_{2}$ transition-metal oxides and dichalcogenides in a honeycomb-like structure. J. Phys. Chem. C 116(16), 8983-8999 (2012). doi:10. $1021 / \mathrm{jp} 212558 \mathrm{p}$

25. A. Splendiani, L. Sun, Y. Zhang, T. Li, J. Kim, C.-Y. Chim, G. Galli, F. Wang, Emerging photoluminescence in monolayer $\mathrm{MoS}_{2}$. Nano Lett. 10(4), 1271-1275 (2010). doi:10.1021/n1903868w

26. K.F. Mak, C. Lee, J. Hone, J. Shan, T.F. Heinz, Atomically thin $\mathrm{MoS}_{2}$ : a new direct-gap semiconductor. Phys. Rev. Lett. 105(13), 136805 (2010). doi:10.1103/PhysRevLett.105.136805

27. A.R. Klots, A.K.M. Newaz, B. Wang, D. Prasai, H. Krzyzanowska et al., Probing excitonic states in suspended two-dimensional semiconductors by photocurrent spectroscopy. Sci. Rep. 4, 6608 (2014). doi:10.1038/srep06608

28. F.A. Rasmussen, K.S. Thygesen, Computational 2D materials database: electronic structure of transition-metal dichalcogenides and oxides. J. Phys. Chem. C 119(23), 13169-13183 (2015). doi:10.1021/acs.jpcc.5b02950

29. K.S. Novoselov, D. Jiang, F. Schedin, T.J. Booth, V.V. Khotkevich, S.V. Morozov, A.K. Geim, Two-dimensional atomic crystals. Proc. Natl. Acad. Sci. 102(30), 10451-10453 (2005). doi:10.1073/pnas.0502848102

30. B. Radisavljevic, A. Radenovic, J. Brivio, V. Giacometti, A. Kis, Single-layer $\mathrm{MoS}_{2}$ transistors. Nat. Nanotechnol. 6(3), 147-150 (2011). doi:10.1038/nnano.2010.279

31. M.S. Fuhrer, J. Hone, Measurement of mobility in dual-gated $\mathrm{MoS}_{2}$ transistors. Nat. Nanotechnol. 8(3), 146-147 (2013). doi:10.1038/nnano.2013.30

32. B. Radisavljevic, A. Kis, Reply to measurement of mobility in dual-gated $\mathrm{MoS}_{2}$ transistors. Nat. Nanotechnol. 8(3), 147-148 (2013). doi:10.1038/nnano.2013.31

33. Z. Yu, Z.-Y. Ong, Y. Pan, Y. Cui, R. Xin et al., Realization of room-temperature phonon-limited carrier transport in monolayer $\mathrm{MoS}_{2}$ by dielectric and carrier screening. Adv. Mater. 28(3), 547-552 (2016). doi:10.1002/adma.201503033

34. X. Li, J.T. Mullen, Z. Jin, K.M. Borysenko, M.B. Nardelli, K.W. Kim, Intrinsic electrical transport properties of monolayer silicene and $\mathrm{MoS}_{2}$ from first principles. Phys. Rev. B 87(11), 115418 (2013). doi:10.1103/PhysRevB.87.115418

35. J. Qiao, X. Kong, Z.-X. Hu, F. Yang, W. Ji, High-mobility transport anisotropy and linear dichroism in few-layer black phosphorus. Nat. Commun. 5, 1-7 (2014). doi:10.1038/ ncomms5475

36. L. Li, Y. Yu, G.J. Ye, Q. Ge, X. Ou, H. Wu, D. Feng, X.H. Chen, Y. Zhang, Black phosphorus field-effect transistors. Nat. Nanotechnol. 9(5), 372-377 (2014). doi:10.1038/nnano. 2014.35

37. W. Zhu, S. Park, M.N. Yogeesh, K.M. McNicholas, S.R. Bank, D. Akinwande, Black phosphorus flexible thin film transistors at gighertz frequencies. Nano Lett. 16(4), 2301-2306 (2016). doi:10.1021/acs.nanolett.5b04768

38. M.E. Davis, J.E. Zuckerman, C.H.J. Choi, D. Seligson, A. Tolcher, C.A. Alabi, Y. Yen, J.D. Heidel, A. Ribas, Evidence of RNAi in humans from systemically administered siRNA via targeted nanoparticles. Nature 464(7291), 1067-1070 (2010). doi: 10.1038/nature08956

39. J. Zou, J. Liu, A.S. Karakoti, A. Kumar, D. Joung, Q. Li, S.I. Khondaker, S. Seal, L. Zhai, Ultralight multiwalled carbon nanotube aerogel. ACS Nano 4(12), 7293-7302 (2010). doi:10. 1021/nn102246a

40. A.S. Karakoti, O. Tsigkou, S. Yue, P.D. Lee, M.M. Stevens, J.R. Jones, S. Seal, Rare earth oxides as nanoadditives in 3-D nanocomposite scaffolds for bone regeneration. J. Mater. Chem. 20(40), 8912-8919 (2010). doi:10.1039/C0JM01072C

41. J.N. Tiwari, R.N. Tiwari, K.S. Kim, Zero-dimensional, one-dimensional, two-dimensional and three-dimensional nanostructured materials for advanced electrochemical energy devices. 
Prog. Mater. Sci. 57(4), 724-803 (2012). doi:10.1016/j.pmatsci. 2011.08.003

42. R. Mas Balleste, C. Gomez Navarro, J. Gomez Herrero, F. Zamora, 2D materials: to graphene and beyond. Nanoscale 3(1), 20-30 (2011). doi:10.1039/C0NR00323A

43. K.S. Novoselov, A.K. Geim, S.V. Morozov, D. Jiang, M.I. Katsnelson, I.V. Grigorieva, S.V. Dubonos, A.A. Firsov, Twodimensional gas of massless Dirac fermions in graphene. Nature 438(7065), 197-200 (2005). doi:10.1038/nature04233

44. E. Canadell, A. LeBeuze, M.A. El Khalifa, R. Chevrel, M.H. Whangbo, Origin of metal clustering in transition-metal chalcogenide layers $\mathrm{MX}_{2}(\mathrm{M}=\mathrm{Nb}, \mathrm{Ta}, \mathrm{Mo}, \mathrm{Re} ; \mathrm{X}=\mathrm{S}, \mathrm{Se})$. J. Am. Chem. Soc. 111(11), 3778-3782 (1989). doi:10.1021/ ja00193a002

45. M.A. Lukowski, A.S. Daniel, C.R. English, F. Meng, A. Forticaux, R.J. Hamers, S. Jin, Highly active hydrogen evolution catalysis from metallic $\mathrm{WS}_{2}$ nanosheets. Energy Environ. Sci. 7(8), 2608-2613 (2014). doi:10.1039/C4EE01329H

46. R.I. Woodward, R.C.T. Howe, T.H. Runcorn, G. Hu, F. Torrisi, E.J.R. Kelleher, T. Hasan, Wideband saturable absorption in few-layer molybdenum diselenide $\left(\mathrm{MoSe}_{2}\right)$ for Q-switching $\mathrm{Yb}$ , Er- and Tm-doped fiber lasers. Opt. Express 23(15), 20051-20061 (2015). doi:10.1364/OE.23.020051

47. J. Wilson, A. Yoffe, The transition metal dichalcogenides discussion and interpretation of the observed optical, electrical and structural properties. Adv. Phys. 18(73), 193-335 (1969). doi:10.1080/00018736900101307

48. E. Singh, K.S. Kim, G.Y. Yeom, H.S. Nalwa, Two-dimensional transition metal dichalcogenide-based counter electrodes for dye-sensitized solar cells. RSC Adv. 7(45), 28234-28290 (2017). doi:10.1039/C7RA03599C

49. P. May, U. Khan, J.N. Coleman, Reinforcement of metal with liquid-exfoliated inorganic nano-platelets. Appl. Phys. Lett. 103(16), 163106 (2013). doi:10.1063/1.4825279

50. W. Choi, N. Choudhary, G.H. Han, J. Park, D. Akinwande, Y.H. Lee, Recent development of two-dimensional transition metal dichalcogenides and their applications. Mater. Today 20(3), 116-130 (2017). doi:10.1016/j.mattod.2016.10.002

51. R. Kappera, D. Voiry, S.E. Yalcin, B. Branch, G. Gupta, A.D. Mohite, M. Chhowalla, Phase-engineered low-resistance contacts for ultrathin $\mathrm{MoS}_{2}$ transistors. Nat. Mater. 13(12), 1128-1134 (2014). doi: $10.1038 /$ nmat4080

52. A. Yoon, Z. Lee, Synthesis and properties of two dimensional doped transition metal dichalcogenides. Appl. Microsc. 47(1), 19-28 (2017). doi:10.9729/AM.2017.47.1.19

53. R. Kappera, D. Voiry, S.E. Yalcin, W. Jen, M. Acerce et al., Metallic 1T phase source/drain electrodes for field effect transistors from chemical vapor deposited $\mathrm{MoS}_{2}$. APL Mater. 2(9), 092516 (2014). doi:10.1063/1.4896077

54. G.L. Frey, S. Elani, M. Homyonfer, Y. Feldman, R. Tenne, Optical-absorption spectra of inorganic fullerenelike $\mathrm{MS}_{2}$ ( $M=$ Mo, W). Phys. Rev. B 57(11), 6666-6671 (1998). doi:10. 1103/PhysRevB.57.6666

55. F. Ji, X. Ren, X. Zheng, Y. Liu, L. Pang, J. Jiang, S.F. Liu, 2D$\mathrm{MoO}_{3}$ nanosheets for superior gas sensors. Nanoscale 8(16), 8696-8703 (2016). doi:10.1039/C6NR00880A

56. J. Wang, C.J. Liu, Preparation of $2 \mathrm{D} \mathrm{WO}_{3}$ Nanomaterials with enhanced catalytic activities: current status and perspective. ChemBioEng Rev. 2(5), 335-350 (2015). doi:10.1002/cben. 201500014

57. S.-J. Choi, J.-S. Jang, H.J. Park, I.-D. Kim, Optically sintered 2D $\mathrm{RuO}_{2}$ nanosheets: temperature-controlled $\mathrm{NO}_{2}$ reaction. Adv. Funct. Mater. 27(13), 1606026 (2017). doi:10.1002/adfm.201606026

58. Y. Zhang, W. Wu, K. Zhang, C. Liu, A. Yu, M. Peng, J. Zhai, Raman study of 2D anatase $\mathrm{TiO}_{2}$ nanosheets. Phys. Chem. Chem. Phys. 18(47), 32178-32184 (2016). doi:10.1039/C6CP05496J
59. Z. Liu, K. Xu, P. She, S. Yin, X. Zhu, H. Sun, Self-assembly of 2D $\mathrm{MnO}_{2}$ nanosheets into high-purity aerogels with ultralow density. Chem. Sci. 7(3), 1926-1932 (2016). doi:10.1039/ C5SC03217B

60. G. Yang, H. Song, M. Wu, C. Wang, $\mathrm{SnO}_{2}$ nanoparticles anchored on $2 \mathrm{D} \mathrm{V}_{2} \mathrm{O}_{5}$ nanosheets with enhanced lithium-storage performances. Electrochim. Acta 205, 153-160 (2016). doi:10. 1016/j.electacta.2016.04.077

61. X. Xu, K. Takada, K. Fukuda, T. Ohnishi, K. Akatsuka, M. Osada, B.T. Hang, K. Kumagai, T. Sekiguchi, T. Sasaki, Tantalum oxide nanomesh as self-standing one nanometre thick electrolyte. Energy Environ. Sci. 4(9), 3509-3512 (2011). doi:10.1039/c1ee01389k

62. G. Eda, T. Fujita, H. Yamaguchi, D. Voiry, M. Chen, M. Chhowalla, Coherent atomic and electronic heterostructures of single-layer $\mathrm{MoS}_{2}$. ACS Nano 6(8), 7311-7317 (2012). doi:10. 1021/nn302422x

63. H. Li, G. Lu, Z. Yin, Q. He, H. Li, Q. Zhang, H. Zhang, Optical identification of single- and few-layer $\mathrm{MoS}_{2}$ sheets. Small 8(5), 682-686 (2012). doi:10.1002/smll.201101958

64. S. Tongay, J. Zhou, C. Ataca, K. Lo, T.S. Matthews, J. Li, J.C. Grossman, J. Wu, Thermally driven crossover from indirect toward direct bandgap in 2D semiconductors: $\mathrm{MoSe}_{2}$ versus $\mathrm{MoS}_{2}$. Nano Lett. 12(11), 5576-5580 (2012). doi:10.1021/ nl302584w

65. K.F. Mak, J. Shan, Photonics and optoelectronics of 2D semiconductor transition metal dichalcogenides. Nat. Photon. 10(4), 216-226 (2016). doi:10.1038/nphoton.2015.282

66. M. Osada, T. Sasaki, Two-dimensional dielectric nanosheets: novel nanoelectronics from nanocrystal building blocks. Adv. Mater. 24(2), 210-228 (2012). doi:10.1002/adma.201103241

67. R.A. Gordon, D. Yang, E.D. Crozier, D.T. Jiang, R.F. Frindt, Structures of exfoliated single layers of $\mathrm{WS}_{2}, \mathrm{MoS}_{2}$, and $\mathrm{MoSe}_{2}$ in aqueous suspension. Phys. Rev. B 65(12), 125407 (2002). doi:10.1103/PhysRevB.65.125407

68. F.R. Gamble, J.H. Osiecki, M. Cais, R. Pisharody, F.J. DiSalvo, T.H. Geballe, Intercalation complexes of lewis bases and layered sulfides: a large class of new superconductors. Science 174(4008), 493-497 (1971). doi:10.1126/Science174.4008.493

69. D.H. Keum, S. Cho, J.H. Kim, D.-H. Choe, H.-J. Sung et al., Bandgap opening in few-layered monoclinic $\mathrm{MoTe}_{2}$. Nat. Phys. 11(6), 482-486 (2015). doi:10.1038/nphys 3314

70. T.A. Empante, Y. Zhou, V. Klee, A.E. Nguyen, I.H. Lu et al., Chemical vapor deposition growth of few-layer $\mathrm{MoTe}_{2}$ in the $2 \mathrm{H}, 1 \mathrm{~T}^{\prime}$, and 1T phases: tunable properties of $\mathrm{MoTe}_{2}$ films. ACS Nano 11(1), 900-905 (2017). doi:10.1021/acsnano.6b07499

71. A.N. Enyashin, L. Yadgarov, L. Houben, I. Popov, M. Weidenbach, R. Tenne, M. Bar-Sadan, G. Seifert, New route for stabilization of $1 \mathrm{~T}-\mathrm{WS}_{2}$ and $\mathrm{MoS}_{2}$ phases. J. Phys. Chem. C 115(50), 24586-24591 (2011). doi:10.1021/jp2076325

72. D. Kong, W. Dang, J.J. Cha, H. Li, S. Meister, H. Peng, Z. Liu, Y. Cui, Few-layer nanoplates of $\mathrm{Bi}_{2} \mathrm{Se}_{3}$ and $\mathrm{Bi}_{2} \mathrm{Te}_{3}$ with highly tunable chemical potential. Nano Lett. 10(6), 2245-2250 (2010). doi:10.1021/nl101260j

73. S. Yang, Y. Gong, Z. Liu, L. Zhan, D.P. Hashim, L. Ma, R. Vajtai, P.M. Ajayan, Bottom-up approach toward single-crystalline $\mathrm{VO}_{2^{-}}$ graphene ribbons as cathodes for ultrafast lithium storage. Nano Lett. 13(4), 1596-1601 (2013). doi:10.1021/nl400001u

74. J. Taha-Tijerina, T.N. Narayanan, G. Gao, M. Rohde, D.A. Tsentalovich, M. Pasquali, P.M. Ajayan, Electrically insulating thermal nano-oils using 2D fillers. ACS Nano 6(2), 1214-1220 (2012). doi:10.1021/nn203862p

75. Y. Ebina, T. Sasaki, M. Harada, M. Watanabe, Restacked perovskite nanosheets and their Pt-loaded materials as photocatalysts. Chem. Mater. 14(10), 4390-4395 (2002). doi:10.1021/ $\mathrm{cm} 020622 \mathrm{e}$ 
76. T.C. Ozawa, K. Fukuda, K. Akatsuka, Y. Ebina, T. Sasaki, Preparation and characterization of the $\mathrm{Eu}^{3+}$ doped perovskite nanosheet phosphor: $\mathrm{La}_{0.90} \mathrm{Eu}_{0.05} \mathrm{Nb}_{2} \mathrm{O}_{7}$. Chem. Mater. 19(26), 6575-6580 (2007). doi:10.1021/cm702552p

77. W.-T. Hsu, Z.-A. Zhao, L.-J. Li, C.-H. Chen, M.-H. Chiu, P.-S. Chang, Y.-C. Chou, W.-H. Chang, Second harmonic generation from artificially stacked transition metal dichalcogenide twisted bilayers. ACS Nano 8(3), 2951-2958 (2014). doi:10.1021/ nn500228r

78. A. Acun, B. Poelsema, H.J.W. Zandvliet, R. van Gastel, The instability of silicene on Ag (111). Appl. Phys. Lett. 103(26), 263119 (2013). doi:10.1063/1.4860964

79. L. Tao, E. Cinquanta, D. Chiappe, C. Grazianetti, M. Fanciulli, M. Dubey, A. Molle, D. Akinwande, Silicene field-effect transistors operating at room temperature. Nat. Nanotechnol. 10(3), 227-231 (2015). doi:10.1038/nnano.2014.325

80. H. Oughaddou, B. Aufray, J.P. Bibérian, J.Y. Hoarau, Growth mode and dissolution kinetics of germanium thin films on $\mathrm{Ag}(001)$ surface: an AES-LEED investigation. Surf. Sci. 429(1), 320-326 (1999). doi:10.1016/S0039-6028(99)00394-5

81. E. Golias, E. Xenogiannopoulou, D. Tsoutsou, P. Tsipas, S.A. Giamini, A. Dimoulas, Surface electronic bands of submonolayer Ge on $\operatorname{Ag}(111)$. Phys. Rev. B 88(7), 075403 (2013). doi:10.1103/PhysRevB.88.075403

82. B. Hao, Y. Yan, X. Wang, G. Chen, Synthesis of anatase $\mathrm{TiO}_{2}$ nanosheets with enhanced pseudocapacitive contribution for fast Lithium storage. ACS Appl. Mater. Interfaces. 5(13), 6285-6291 (2013). doi:10.1021/am4013215

83. M. Sterrer, T. Risse, U. Martinez Pozzoni, L. Giordano, M. Heyde, H.-P. Rust, G. Pacchioni, H.-J. Freund, Control of the charge state of metal atoms on thin $\mathrm{MgO}$ films. Phys. Rev. Lett. 98(9), 096107 (2007). doi:10.1103/PhysRevLett.98. 096107

84. H. Jung, J. Park, I.-K. Oh, T. Choi, S. Lee, J. Hong, T. Lee, S.-H. Kim, H. Kim, Fabrication of transferable $\mathrm{Al}_{2} \mathrm{O}_{3}$ nanosheet by atomic layer deposition for graphene FET. ACS Appl. Mater. Interfaces 6(4), 2764-2769 (2014). doi:10.1021/am4052987

85. K.S. Novoselov, A.K. Geim, S.V. Morozov, D. Jiang, Y. Zhang, S.V. Dubonos, I.V. Grigorieva, A.A. Firsov, Electric field effect in atomically thin carbon films. Science 306(5696), 666-669 (2004). doi:10.1126/Science 1102896

86. B.K. Miremadi, S.R. Morrison, High activity catalyst from exfoliated $\mathrm{MoS}_{2}$. J. Catalysis 103(2), 334-345 (1987). doi:10. 1016/0021-9517(87)90125-4

87. D.J. Late, Temperature dependent phonon shifts in single-layer $\mathrm{WS}_{2}$. ACS Appl. Mater. Interfaces. 6(2), 1158-1163 (2014). doi:10.1021/am404847d

88. P. De Padova, C. Ottaviani, C. Quaresima, B. Olivieri, P. Imperatori et al., $24 \mathrm{~h}$ stability of thick multilayer silicene in air. 2D Mater. 1(2), 1003 (2014). doi:10.1088/2053-1583/1/2/021003

89. J.N. Coleman, M. Lotya, A.O. Neill, S.D. Bergin, P.J. King et al., Two-dimensional nanosheets produced by liquid exfoliation of layered materials. Science 331(6017), 568-571 (2011). doi:10.1126/Science 1194975

90. K.G. Zhou, N.-N. Mao, H.-X. Wang, Y. Peng, H.-L. Zhang, A mixed-solvent strategy for efficient exfoliation of inorganic graphene analogues. Angew. Chem. Int. Ed. 50(46), 10839-10842 (2011). doi:10.1002/anie.201105364

91. R.J. Smith, P.J. King, M. Lotya, C. Wirtz, U. Khan et al., Largescale exfoliation of inorganic layered compounds in aqueous surfactant solutions. Adv. Mater. 23(34), 3944-3948 (2011). doi:10.1002/adma.201102584

92. B.C. Brodie, On the atomic weight of graphite. Philos. Trans. R. Soc. London 149, 249-259 (1859). doi:10.1098/rstl.1859.0013

93. V.H. Luan, H.N. Tien, L.T. Hoa, N.T.M. Hien, E.-S. Oh et al., Synthesis of a highly conductive and large surface area graphene oxide hydrogel and its use in a supercapacitor. J. Mater. Chem. A 1(2), 208-211 (2013). doi:10.1039/C2TA00444E

94. W.S. Hummers, R.E. Offeman, Preparation of graphitic oxide. J. Am. Chem. Soc. 80(6), 1339 (1958). doi:10.1021/ja01539 a017

95. G. Goncalves, P.A.A.P. Marques, C.M. Granadeiro, H.I.S. Nogueira, M.K. Singh, J. Grácio, Surface modification of graphene nanosheets with gold nanoparticles: the role of oxygen moieties at graphene surface on gold nucleation and growth. Chem. Mater. 21(20), 4796-4802 (2009). doi:10.1021/cm9010 $52 \mathrm{~s}$

96. F. Xiong, H. Wang, X. Liu, J. Sun, M. Brongersma, E. Pop, Y. Cui, Li Intercalation in $\mathrm{MoS}_{2}$ : in Situ observation of its dynamics and tuning optical and electrical properties. Nano Lett. 15(10), 6777-6784 (2015). doi:10.1021/acs.nanolett. 5 b02619

97. M. Petrović, I. Šrut Rakić, S. Runte, C. Busse, J.T. Sadowski et al., The mechanism of caesium intercalation of graphene. Nat. Commun. 4, 1-10 (2013). doi:10.1038/ncomms 3772

98. V. Nicolosi, M. Chhowalla, M.G. Kanatzidis, M.S. Strano, J.N. Coleman, Liquid exfoliation of layered materials. Science 340(6139), 1226419 (2013). doi:10.1126/Science1226419

99. Y. Zhan, Z. Liu, S. Najmaei, P.M. Ajayan, J. Lou, Large-area vapor-phase growth and characterization of $\mathrm{MoS}_{2}$ atomic layers on a $\mathrm{SiO}_{2}$ substrate. Small 8(7), 966-971 (2012). doi:10.1002/ smll.201102654

100. Y.H. Lee, X.Q. Zhang, W. Zhang, M.T. Chang, C.T. Lin et al., Synthesis of large-area $\mathrm{MoS}_{2}$ atomic layers with chemical vapor deposition. Adv. Mater. 24(17), 2320-2325 (2012). doi:10.1002/ adma.201104798

101. K.K. Liu, W. Zhang, Y.H. Lee, Y.C. Lin, M.T. Chang et al., Growth of large-area and highly crystalline $\mathrm{MoS}_{2}$ thin layers on insulating substrates. Nano Lett. 12(3), 1538-1544 (2012). doi: $10.1021 / \mathrm{nl} 2043612$

102. E.S. Peters, C.J. Carmalt, I.P. Parkin, Dual-source chemical vapour deposition of titanium sulfide thin films from tetrakisdimethylamidotitanium and sulfur precursors. J. Mater. Chem. 14(23), 3474-3477 (2004). doi:10.1039/B410390D

103. N.D. Boscher, C.S. Blackman, C.J. Carmalt, I.P. Parkin, A.G. Prieto, Atmospheric pressure chemical vapour deposition of vanadium diselenide thin films. Appl. Surf. Sci. 253(14), 6041-6046 (2007). doi:10.1016/j.apsusc.2007.01.002

104. N.D. Boscher, C.J. Carmalt, I.P. Parkin, Atmospheric pressure chemical vapor deposition of $\mathrm{WSe}_{2}$ thin films on glass-highly hydrophobic sticky surfaces. J. Mater. Chem. 16(1), 122-127 (2006). doi:10.1039/B514440J

105. C.J. Carmalt, I.P. Parkin, E.S. Peters, Atmospheric pressure chemical vapour deposition of $\mathrm{WS}_{2}$ thin films on glass. Polyhedron 22(11), 1499-1505 (2003). doi:10.1016/S02775387(03)00194-3

106. N.D. Boscher, C.J. Carmalt, R.G. Palgrave, J.J. Gil-Tomas, I.P. Parkin, Atmospheric pressure CVD of molybdenum diselenide films on glass. Chem. Vapor Depos. 12(11), 692-698 (2006). doi:10.1002/cvde. 200606502

107. F. Müller, K. Stöwe, H. Sachdev, Symmetry versus commensurability: epitaxial growth of hexagonal boron nitride on $\mathrm{Pt}$ (111) from B-trichloroborazine $(\mathrm{ClBNH}) 3$. Chem. Mater. 17(13), 3464-3467 (2005). doi:10.1021/cm048629e

108. W. Auwärter, H.U. Suter, H. Sachdev, T. Greber, Synthesis of one monolayer of hexagonal boron nitride on $\mathrm{Ni}(111)$ from B-trichloroborazine $(\mathrm{ClBNH})_{3}$. Chem. Mater. 16(2), 343-345 (2004). doi:10.1021/cm034805s

109. A. Nagashima, N. Tejima, Y. Gamou, T. Kawai, C. Oshima, Electronic states of monolayer hexagonal boron nitride formed on the metal surfaces. Surf. Sci. 357, 307-311 (1996). doi:10. 1016/0039-6028(96)00134-3 
110. Y. Shi, W. Zhou, A.-Y. Lu, W. Fang, Y.-H. Lee et al., Van der Waals epitaxy of $\mathrm{MoS}_{2}$ layers using graphene as growth templates. Nano Lett. 12(6), 2784-2791 (2012). doi:10.1021/ $\mathrm{nl} 204562 \mathrm{j}$

111. Y. Zheng, A. Koëbel, J.F. Pétroff, J.C. Boulliard, B. Capelle, M. Eddrief, GaSeSi (111) heteroepitaxy: the early stages of growth. J. Cryst. Growth 162(3), 135-141 (1996). doi:10.1016/00220248(95)00952-3

112. B. Lalmi, H. Oughaddou, H. Enriquez, A. Kara, S. Vizzini, B. Ealet, B. Aufray, Epitaxial growth of a silicene sheet. Appl. Phys. Lett. 97(22), 223109 (2010). doi:10.1063/1.3524215

113. C. Léandri, H. Oughaddou, B. Aufray, J.M. Gay, G. Le Lay, A. Ranguis, Y. Garreau, Growth of Si nanostructures on $\mathrm{Ag}(001)$. Surf. Sci. 601(1), 262-267 (2007). doi:10.1016/j.susc.2006.09. 030

114. B. Aufray, A. Kara, S. Vizzini, H. Oughaddou, C. Léandri, B. Ealet, G. Le Lay, Graphene-like silicon nanoribbons on $\mathrm{Ag}(110)$ : a possible formation of silicene. Appl. Phys. Lett. 96(18), 183-192 (2010). doi:10.1063/1.3419932

115. P. De Padova, O. Kubo, B. Olivieri, C. Quaresima, T. Nakayama, M. Aono, G. Le Lay, Multilayer silicene nanoribbons. Nano Lett. 12(11), 5500-5503 (2012). doi:10.1021/ nl302598x

116. H. Oughaddou, J.M. Gay, B. Aufray, L. Lapena, G. Le Lay, O. Bunk, G. Falkenberg, J.H. Zeysing, R.L. Johnson, Ge tetramer structure of the $\mathrm{p}(2 \sqrt{2} \times 4 \sqrt{2}) \mathrm{R} 45^{\circ}$ surface reconstruction of $\mathrm{Ge} / \mathrm{Ag}(001)$ : a surface $\mathrm{x}$-ray diffraction and STM study. Phys. Rev. B 61(8), 5692-5697 (2000). doi:10.1103/PhysRevB.61. 5692

117. Y. Peng, Z. Meng, C. Zhong, J. Lu, W. Yu, Y. Jia, Y. Qian, Hydrothermal synthesis and characterization of single-molecular-layer $\mathrm{MoS}_{2}$ and $\mathrm{MoSe}_{2}$. Chem. Lett. 30(8), 772-773 (2001). doi:10.1246/cl.2001.772

118. Y. Li, H. Wang, L. Xie, Y. Liang, G. Hong, H. Dai, $\mathrm{MoS}_{2}$ nanoparticles grown on graphene: an advanced catalyst for the hydrogen evolution reaction. J. Am. Chem. Soc. 133(19), 7296-7299 (2011). doi:10.1021/ja201269b

119. S. Jeong, D. Yoo, J.-T. Jang, M. Kim, J. Cheon, Well-defined colloidal 2-D layered transition-metal chalcogenide nanocrystals via generalized synthetic protocols. J. Am. Chem. Soc. 134(44), 18233-18236 (2012). doi:10.1021/ja3089845

120. L. Qiliang, K. Sang-Mo, C.A. Richter, M.D. Edelstein, J.E. Bonevich, J.J. Kopanski, J.S. Suehle, E.M. Vogel, Precise alignment of single nanowires and fabrication of nanoelectromechanical switch and other test structures. IEEE Trans. Nanotechnol. 6(2), 256-262 (2007). doi:10.1109/TNANO.2007. 891827

121. Y.-W. Jun, J.-W. Seo, S.J. Oh, J. Cheon, Recent advances in the shape control of inorganic nano-building blocks. Coordin. Chem. Rev. 249(17), 1766-1775 (2005). doi:10.1016/j.ccr.2004. 12.008

122. Y. Xu, Z. Liu, X. Zhang, Y. Wang, J. Tian, Y. Huang, Y. Ma, X. Zhang, Y. Chen, A graphene hybrid material covalently functionalized with porphyrin: synthesis and optical limiting property. Adv. Mater. 21(12), 1275-1279 (2009). doi:10.1002/adma. 200801617

123. Y. Hernandez, V. Nicolosi, M. Lotya, F.M. Blighe, Z. Sun et al., High-yield production of graphene by liquid-phase exfoliation of graphite. Nat. Nanotechnol. 3(9), 563-568 (2008). doi:10. 1038/nnano.2008.215

124. D.A. Dikin, S. Stankovich, E.J. Zimney, R.D. Piner, G.H.B. Dommett, G. Evmenenko, S.T. Nguyen, R.S. Ruoff, Preparation and characterization of graphene oxide paper. Nature 448(7152), 457-460 (2007). doi:10.1038/nature06016

125. Q. Xiang, J. Yu, M. Jaroniec, Synergetic effect of $\mathrm{MoS}_{2}$ and graphene as cocatalysts for enhanced photocatalytic $\mathrm{H}_{2}$ production activity of $\mathrm{TiO}_{2}$ nanoparticles. J. Am. Chem. Soc. 134(15), 6575-6578 (2012). doi:10.1021/ja302846n

126. G. Wang, X. Shen, J. Yao, J. Park, Graphene nanosheets for enhanced lithium storage in lithium ion batteries. Carbon 47(8), 2049-2053 (2009). doi:10.1016/j.Carbon2009.03.053

127. G. Zhou, F. Li, H.-M. Cheng, Progress in flexible lithium batteries and future prospects. Energy Environ. Sci. 7(4), 1307-1338 (2014). doi:10.1039/C3EE43182G

128. M.D. Patel, E. Cha, N. Choudhary, C. Kang, W. Lee, J.Y. Hwang, W. Choi, Vertically oriented $\mathrm{MoS}_{2}$ nanoflakes coated on 3D carbon nanotubes for next generation Li-ion batteries. Nanotechnology 27(49), 495401 (2016). doi:10.1088/09574484/27/49/495401

129. J.J. Yoo, K. Balakrishnan, J. Huang, V. Meunier, B.G. Sumpter et al., Ultrathin planar graphene supercapacitors. Nano Lett. 11(4), 1423-1427 (2011). doi:10.1021/n1200225j

130. A. Mhamdi, E.B. Salem, S. Jaziri, Electronic reflection for a single-layer graphene quantum well. Solid State Commun. 175, 106-113 (2013). doi:10.1016/j.ssc.2013.04.026

131. A.K. Geim, Graphene: status and prospects. Science 324(5934), 1530-1534 (2009). doi:10.1126/Science1158877

132. Y. Zhang, T.-T. Tang, C. Girit, Z. Hao, M.C. Martin, A. Zettl, M.F. Crommie, Y.R. Shen, F. Wang, Direct observation of a widely tunable bandgap in bilayer graphene. Nature 459(7248), 820-823 (2009). doi:10.1038/nature08105

133. T.M.G. Mohiuddin, A. Lombardo, R.R. Nair, A. Bonetti, G. Savini et al., Uniaxial strain in graphene by raman spectroscopy: $\mathrm{G}$ peak splitting, gruneisen parameters, and sample orientation. Phys. Rev. B 79(20), 205433 (2009). doi:10.1103/PhysRevB.79. 205433

134. H.J. Conley, B. Wang, J.I. Ziegler, R.F. Haglund, S.T. Pantelides, K.I. Bolotin, Bandgap engineering of strained monolayer and bilayer $\mathrm{MoS}_{2}$. Nano Lett. 13(8), 3626-3630 (2013). doi:10. $1021 / \mathrm{nl} 4014748$

135. X. Li, X. Wang, L. Zhang, S. Lee, H. Dai, Chemically derived, ultrasmooth graphene nanoribbon semiconductors. Science 319(5867), 1229-1232 (2008). doi:10.1126/Science1150878

136. R. Akis, D.K. Ferry, Using magnetic fields and band gap engineering to achieve robust spin filtering in finite quantum dot arrays. J. Phys: Conf. Ser. 109(1), 012005 (2008). doi:10.1088/ 1742-6596/109/1/012005

137. J.C. Meyer, A.K. Geim, M.I. Katsnelson, K.S. Novoselov, T.J. Booth, S. Roth, The structure of suspended graphene sheets. Nature 446(7131), 60-63 (2007). doi:10.1038/nature05545

138. X. Du, I. Skachko, A. Barker, E.Y. Andrei, Approaching ballistic transport in suspended graphene. Nat. Nanotechnol. 3(8), 491-495 (2008). doi:10.1038/nnano.2008.199

139. K.K. Kam, B.A. Parkinson, Detailed photocurrent spectroscopy of the semiconducting group VIB transition metal dichalcogenides. J. Phys. Chem. 86(4), 463-467 (1982). doi:10.1021/j100393a010

140. K. Uchida, H. Watanabe, A. Kinoshita, J. Koga, T. Numata, S. Takagi, Experimental study on carrier transport mechanism in ultrathin-body SOI nand p-MOSFETs with SOI thickness less than $5 \mathrm{~nm}$. IEEE Int. Electron Devices Meeting (IEDM), pp. 47-50 (2002). doi:10.1109/IEDM.2002.1175776

141. D. Yin, Y. Yoon, Design strategy of two-dimensional material field-effect transistors: engineering the number of layers in phosphorene FETs. J. Appl. Phys. 119(21), 214312 (2016). doi: $10.1063 / 1.4953256$

142. T.F. Chung, T. Shen, H. Cao, A. Luis, W. Wu, Q. Yu, D. Newell, Y.P. Chen, Synthetic graphene grown by chemical vapor deposition on copper foils. Inter. J. Mod. Phys. B 27(10), 1341002 (2013). doi:10.1142/S0217979213410026

143. K. Kalantar-Zadeh, J.Z. Ou, Biosensors based on two-dimensional $\mathrm{MoS}_{2}$. ACS Sens. 1(1), 5-16 (2016). doi:10.1021/acssen sors.5b00142 
144. A. Reina, X. Jia, J. Ho, D. Nezich, H. Son, V. Bulovic, M.S. Dresselhaus, J. Kong, Large area, few-layer graphene films on arbitrary substrates by chemical vapor deposition. Nano Lett. 9(1), 30-35 (2009). doi: $10.1021 / \mathrm{nl} 801827 \mathrm{v}$

145. N. Mao, Y. Chen, D. Liu, J. Zhang, L. Xie, Solvatochromic effect on the photoluminescence of $\mathrm{MoS}_{2}$ monolayers. Small 9(8), 1312-1315 (2013). doi:10.1002/smll.201202982

146. H. Zhang, Q. Bao, D. Tang, L. Zhao, K. Loh, Large energy soliton erbium-doped fiber laser with a graphene-polymer composite mode locker. Appl. Phys. Lett. 95(14), 141103 (2009). doi:10.1063/1.3244206

147. S. Lu, C. Zhao, Y. Zou, S. Chen, Y. Chen, Y. Li, H. Zhang, S. Wen, D. Tang, Third order nonlinear optical property of $\mathrm{Bi}_{2} \mathrm{Se}_{3}$. Opt. Express 21(2), 2072-2082 (2013). doi:10.1364/OE.21. 002072

148. H. Zhang, S.B. Lu, J. Zheng, J. Du, S.C. Wen, D.Y. Tang, K.P. Loh, Molybdenum disulfide $\left(\mathrm{MoS}_{2}\right)$ as a broadband saturable absorber for ultra-fast photonics. Opt. Express 22(6), 7249-7260 (2014). doi:10.1364/OE.22.007249

149. S.B. Lu, L.L. Miao, Z.N. Guo, X. Qi, C.J. Zhao, H. Zhang, S.C. Wen, D.Y. Tang, D.Y. Fan, Broadband nonlinear optical response in multi-layer black phosphorus: an emerging infrared and mid-infrared optical material. Opt. Express 23(9), 11183-11194 (2015). doi:10.1364/OE.23.011183

150. R.R. Nair, P. Blake, A.N. Grigorenko, K.S. Novoselov, T.J. Booth, T. Stauber, N.M.R. Peres, A.K. Geim, Fine structure constant defines visual transparency of graphene. Science 320(5881), 1308 (2008). doi:10.1126/Science1156965

151. G. Eda, G. Fanchini, M. Chhowalla, Large-area ultrathin films of reduced graphene oxide as a transparent and flexible electronic material. Nat. Nanotechnol. 3(5), 270-274 (2008). doi:10.1038/ nature 05545

152. C. Lee, X. Wei, J.W. Kysar, J. Hone, Measurement of the elastic properties and intrinsic strength of monolayer graphene. Science 321(5887), 385-388 (2008). doi:10.1126/Science1157996

153. C. Gómez-Navarro, M. Burghard, K. Kern, Elastic properties of chemically derived single graphene sheets. Nano Lett. 8(7), 2045-2049 (2008). doi:10.1021/n1801384y

154. An Introduction to FETs. Radio Commun. 76(7), 1-5 (2000). http://www.colorado.edu/physics/phys3330/phys3330_sp15/ resources/AN101FETintro.pdf (accessed)

155. I. Ferain, C.A. Colinge, J.-P. Colinge, Multigate transistors as the future of classical metal-oxide-semiconductor field-effect transistors. Nature 479(7373), 310-316 (2011). doi:10.1038/ nature 10676

156. G.D. Wilk, R.M. Wallace, J.M. Anthony, High-к gate dielectrics: current status and materials properties considerations. J. Appl. Phys. 89(10), 5243-5275 (2001). doi:10.1063/1.1361065

157. A.C. Seabaugh, Q. Zhang, Low-voltage tunnel transistors for beyond CMOS logic. Proc. IEEE 98(12), 2095-2110 (2010). doi:10.1109/JPROC.2010.2070470

158. A.M. Ionescu, H. Riel, Tunnel field-effect transistors as energyefficient electronic switches. Nature 479(7373), 329-337 (2011). doi:10.1038/nature10679

159. R.K. Ghosh, S. Mahapatra, Monolayer transition metal dichalcogenide channel-based tunnel transistor. IEEE J. Electron Devices Soc. 1(10), 175-180 (2013). doi:10.1109/JEDS.2013. 2292799

160. Q. Zhang, G. Iannaccone, G. Fiori, Two-dimensional tunnel transistors based on $\mathrm{Bi}_{2} \mathrm{Se}_{3}$ thin film. IEEE Electron Device Lett. 35(1), 129-131 (2014). doi:10.1109/LED.2013.2288036

161. W.G. Song, H.-J. Kwon, J. Park, J. Yeo, M. Kim et al., Highperformance flexible multilayer $\mathrm{MoS}_{2}$ transistors on solutionbased polyimide substrates. Adv. Funct. Mater. 26(15), 2426-2434 (2016). doi:10.1002/adfm.201505019
162. Y.K. Hong, G. Yoo, J. Kwon, S. Hong, W.G. Song et al., High performance and transparent multilayer $\mathrm{MoS}_{2}$ transistors: tuning Schottky barrier characteristics. AIP Adv. 6(5), 055026 (2016). doi:10.1063/1.4953062

163. L. Liu, Y. Lu, J. Guo, On monolayer $\mathrm{MoS}_{2}$ field-effect transistors at the scaling limit. IEEE Trans. Electron Devices 60(12), 4133-4139 (2013). doi:10.1109/TED.2013.2284591

164. L. Kai-Shin, W. Bo-Wei, L. Lain-Jong, L. Ming-Yang, C. ChiaChin Kevin, et al., $\mathrm{MoS}_{2} \mathrm{U}$-shape MOSFET with $10 \mathrm{~nm}$ channel length and poly-Si source/drain serving as seed for full wafer CVD $\mathrm{MoS}_{2}$ availability. In Proc. IEEE Symp. VLSI Technol., pp. 1-2 (2016). doi:10.1109/VLSIT.2016.7573375

165. K.S. Novoselov, V.I. Falko, L. Colombo, P.R. Gellert, M.G. Schwab, K. Kim, A roadmap for graphene. Nature 490(7419), 192-200 (2012). doi:10.1038/nature 11458

166. L. Liu, S.B. Kumar, Y. Ouyang, J. Guo, Performance limits of monolayer transition metal dichalcogenide transistors. IEEE Trans. Electron Devices 58(9), 3042-3047 (2011). doi:10.1109/ TED.2011.2159221

167. Y. Naveh, K. Likharev, Modeling of 10-nm-scale ballistic MOSFET's. IEEE Electron Device Lett. 21(5), 242-244 (2000). doi: $10.1109 / 55.841309$

168. A. Di Bartolomeo, Graphene schottky diodes: an experimental review of the rectifying graphene/semiconductor heterojunction. Phys. Rep. 606, 1-58 (2016). doi:10.1016/j.physrep.2015.10.003

169. A.A. Balandin, S. Ghosh, W. Bao, I. Calizo, D. Teweldebrhan, F. Miao, C.N. Lau, Superior thermal conductivity of single-layer graphene. Nano Lett. 8(3), 902-907 (2008). doi:10.1021/nl0731872

170. I. Jo, M.T. Pettes, J. Kim, K. Watanabe, T. Taniguchi, Z. Yao, L. Shi, Thermal conductivity and phonon transport in suspended few-layer hexagonal boron nitride. Nano Lett. 13(2), 550-554 (2013). doi: $10.1021 / \mathrm{nl} 304060 \mathrm{~g}$

171. S. Ghosh, I. Calizo, D. Teweldebrhan, E.P. Pokatilov, D.L. Nika, A.A. Balandin, W. Bao, F. Miao, C.N. Lau, Extremely high thermal conductivity of graphene: prospects for thermal management applications in nanoelectronic circuits. Appl. Phys. Lett. 92(15), 151911 (2008). doi:10.1063/1.2907977

172. J. Lan, J.-S. Wang, C.K. Gan, S.K. Chin, Edge effects on quantum thermal transport in graphene nanoribbons: tightbinding calculations. Phys. Rev. B 79(11), 115401 (2009). doi:10.1103/PhysRevB.79.115401

173. D.L. Nika, E.P. Pokatilov, A.S. Askerov, A.A. Balandin, Phonon thermal conduction in graphene: role of umklapp and edge roughness scattering. Phys. Rev. B 79(15), 155413 (2009). doi:10.1103/PhysRevB.79.155413

174. Y. Cai, J. Lan, G. Zhang, Y.-W. Zhang, Lattice vibrational modes and phonon thermal conductivity of monolayer $\mathrm{MoS}_{2}$. Phys. Rev. B 89(3), 035438 (2014). doi:10.1103/PhysRevB.89. 035438

175. X. Liu, G. Zhang, Q.-X. Pei, Y.-W. Zhang, Phonon thermal conductivity of monolayer $\mathrm{MoS}_{2}$ sheet and nanoribbons. Appl. Phys. Lett. 103(13), 133113 (2013). doi:10.1063/1.4823509

176. J.-W. Jiang, H.S. Park, T. Rabczuk, Molecular dynamics simulations of single-layer molybdenum disulphide $\left(\mathrm{MoS}_{2}\right)$ : stillinger-weber parametrization, mechanical properties, and thermal conductivity. J. Appl. Phys. 114(6), 064307 (2013). doi:10.1063/ 1.4818414

177. J.-Y. Kim, S.-M. Choi, W.-S. Seo, W.-S. Cho, Thermal and electronic properties of exfoliated metal chalcogenides. Bull. Korean Chem. Soc. 31(11), 3225-3227 (2010). doi:10.5012/ bkcs.2010.31.11.3225

178. S. Sahoo, A.P. Gaur, M. Ahmadi, M.J.-F. Guinel, R.S. Katiyar, Temperature-dependent raman studies and thermal conductivity of few-layer $\mathrm{MoS}_{2}$. J. Phys. Chem. C 117(17), 9042-9047 (2013). doi:10.1021/jp402509w 
179. Z. Zong, L. Li, J. Jang, N. Lu, M. Liu, Analytical surfacepotential compact model for amorphous-IGZO thin-film transistors. J. Appl. Phys. 117(21), 215705 (2015). doi:10.1063/1. 4922181

180. S. Krishnamoorthy, M.H. Chowdhury, Investigation and a practical compact network model of thermal stress in integrated circuits. Integr. Comput.-Aided Eng. 16(2), 131-140 (2009). doi:10.3233/ICA-2009-0310

181. X. Qian, Y. Wang, W. Li, J. Lu, J. Li, Modelling of stacked 2D materials and devices. 2D Mater. 2(3), 032003 (2015). doi:10. 1088/2053-1583/2/3/032003/meta

182. N. Lu, P. Sun, L. Li, Q. Liu, S. Long, L. Hangbing, M. Liu, Thermal effect on endurance performance of 3-dimensional RRAM crossbar array. Chin. Phys. B 25(5), 056501 (2016). doi:10.1088/1674-1056/25/5/056501

183. P. Buccella, C. Stefanucci, H. Zou, Y. Moursy, R. Iskander, J.M. Sallese, M. Kayal, Methodology for 3-D substrate network extraction for spice simulation of parasitic currents in smart power ICs. IEEE Trans. Comput.-Aided Des. Integr. Circuits Syst. 35(9), 1489-1502 (2016). doi:10.1109/TCAD.2015. 2513008

184. L. Nianduan, W. Lingfei, L. Ling, L. Ming, A review for compact model of graphene field-effect transistors. Chin. Phys. B 26(3), 036804 (2017). doi:10.1088/1674-1056/26/3/036804/ meta

185. Y. Yoon, K. Ganapathi, S. Salahuddin, How good can monolayer $\mathrm{MoS}_{2}$ transistors Be. Nano Lett. 11(9), 3768-3773 (2011). doi:10.1021/nl2018178

186. N. Ma, D. Jena, Carrier statistics and quantum capacitance effects on mobility extraction in two-dimensional crystal semiconductor field-effect transistors. 2D Mater. 2(1), 015003 (2015). doi:10.1088/2053-1583/2/1/015003/meta

187. Y. Taur, J. Wu, J. Min, A Short-Channel I-V Model for 2-D MOSFETs. IEEE Trans. Electron Devices 63(6), 2550-2555 (2016). doi:10.1109/TED.2016.2547949

188. D. Jiménez, Drift-diffusion model for single layer transition metal dichalcogenide field-effect transistors. Appl. Phys. Lett. 101(24), 243501 (2012). doi:10.1063/1.4770313

189. C. Kshirsagar, W. Xu, C. Kim, S. Koester, Design and analysis of $\mathrm{MoS}_{2}$-based MOSFETs for ultra-low-leakage dynamic memory applications. In 72nd Annu. Device Res. Conf., pp.187-188 (2014). doi:10.1109/DRC.2014.6872360

190. W. Cao, J. Kang, W. Liu, K. Banerjee, A compact currentvoltage model for 2D semiconductor based field-effect transistors considering interface traps, mobility degradation, and inefficient doping effect. IEEE Trans. Electron Devices 61(12), 4282-4290 (2014). doi:10.1109/TED.2014.2365028

191. C. Yadav, A. Agarwal, Y.S. Chauhan, Compact modeling of transition metal dichalcogenide based thin body transistors and circuit validation. IEEE Trans. Electron Devices 64(3), 1261-1268 (2017). doi:10.1109/TED.2016.2643698

192. F. Schwierz, Graphene transistors. Nat. Nanotechnol. 5(7), 487-496 (2010). doi:10.1038/nnano.2010.89

193. A. Ayari, E. Cobas, O. Ogundadegbe, M.S. Fuhrer, Realization and electrical characterization of ultrathin crystals of layered transition-metal dichalcogenides. J. Appl. Phys. 101(1), 14507 (2007). doi:10.1063/1.2407388

194. B. Radisavljevic, M.B. Whitwick, A. Kis, Correction to integrated circuits and logic operations based on single-layer $\mathrm{MoS}_{2}$. ACS Nano 7(4), 3729 (2013). doi:10.1021/nn400553g

195. O. Lopez-Sanchez, D. Lembke, M. Kayci, A. Radenovic, A. Kis, Ultrasensitive photodetectors based on monolayer $\mathrm{MoS}_{2}$. Nat. Nanotechnol. 8(7), 497-501 (2013). doi:10.1038/nnano.2013.100

196. W.J. Yu, Y. Liu, H. Zhou, A. Yin, Z. Li, Y. Huang, X. Duan, Highly efficient gate-tunable photocurrent generation in vertical heterostructures of layered materials. Nat. Nanotechnol. 8(12), 952-958 (2013). doi:10.1038/nnano.2013.219

197. M.R. Esmaeili-Rad, S. Salahuddin, High performance molybdenum disulfide amorphous silicon heterojunction photodetector. Sci. Rep. 3, 1-6 (2013). doi:10.1038/srep02345

198. B. Liu, M. Fathi, L. Chen, A. Abbas, Y. Ma, C. Zhou, Chemical vapor deposition growth of monolayer $\mathrm{WSe}_{2}$ with tunable device characteristics and growth mechanism study. ACS Nano 9(6), 6119-6127 (2015). doi:10.1021/acsnano.5b01301

199. H. Fang, S. Chuang, T.C. Chang, K. Takei, T. Takahashi, A. Javey, High-performance single layered $\mathrm{WSe}_{2} \mathrm{p}$-FETs with chemically doped contacts. Nano Lett. 12(7), 3788-3792 (2012). doi: $10.1021 / \mathrm{nl} 301702 \mathrm{r}$

200. V. Podzorov, M. Gershenson, C. Kloc, R. Zeis, E. Bucher, Highmobility field-effect transistors based on transition metal dichalcogenides. Appl. Phys. Lett. 84(17), 3301-3303 (2004). doi:10.1063/1.1723695

201. J.-K. Huang, J. Pu, C.-L. Hsu, M.-H. Chiu, Z.-Y. Juang et al., Large-area synthesis of highly crystalline $\mathrm{WSe}_{2}$ monolayers and device applications. ACS Nano 8(1), 923-930 (2014). doi:10. 1021/nn405719x

202. J.C. Bernède, M. Kettaf, A. Khelil, M. Spiesser, p-n junctions in molybdenum ditelluride. Phys. Status Solidi A 157(1), 205-209 (1996). doi:10.1002/pssa.2211570126

203. A. Conan, A. Bonnet, M. Zoaeter, D. Ramoul, Dependence of the total mobility in a one-band model applicationto n-type $\mathrm{MoTe}_{2}$. Phys. Status Solidi B 124(1), 403-410 (1984). doi:10. 1002/pssb.2221240144

204. Y.-F. Lin, Y. Xu, C.-Y. Lin, Y.-W. Suen, M. Yamamoto, S. Nakaharai, K. Ueno, K. Tsukagoshi, Origin of noise in layered $\mathrm{MoTe}_{2}$ transistors and its possible use for environmental sensors. Adv. Mater. 27(42), 6612-6619 (2015). doi:10.1002/adma. 201502677

205. Y.-F. Lin, Y. Xu, S.-T. Wang, S.-L. Li, M. Yamamoto et al., Ambipolar $\mathrm{MoTe}_{2}$ transistors and their applications in logic circuits. Adv. Mater. 26(20), 3263-3269 (2014). doi:10.1002/ adma.201305845

206. H. Liu, A.T. Neal, P.D. Ye, Channel length scaling of $\mathrm{MoS}_{2}$ MOSFETs. ACS Nano 6(10), 8563-8569 (2012). doi:10.1021/ nn303513c

207. S. Ghatak, A.N. Pal, A. Ghosh, Nature of electronic states in atomically thin $\mathrm{MoS}_{2}$ field-effect transistors. ACS Nano 5(10), 7707-7712 (2011). doi:10.1021/nn202852j

208. H. Liu, P.D. Ye, $\mathrm{MoS}_{2}$ dual-gate MOSFET with atomiclayer-deposited $\mathrm{Al}_{2} \mathrm{O}_{3}$ as top-gate dielectric. IEEE Electron Device Lett. 33(4), 546-548 (2012). doi:10.1109/LED.2012. 2184520

209. K. Lee, H.-Y. Kim, M. Lotya, J.N. Coleman, G.-T. Kim, G.S. Duesberg, Electrical characteristics of molybdenum disulfide flakes produced by liquid exfoliation. Adv. Mater. 23(36), 4178-4182 (2011). doi:10.1002/adma.201101013

210. M.M. Perera, M.-W. Lin, H.-J. Chuang, B.P. Chamlagain, C. Wang, X. Tan, M.M.-C. Cheng, D. Tománek, Z. Zhou, Improved carrier mobility in few-layer $\mathrm{MoS}_{2}$ field-effect transistors with ionic-liquid gating. ACS Nano 7(5), 4449-4458 (2013). doi:10.1021/nn401053g

211. G.-H. Lee, Y.-J. Yu, X. Cui, N. Petrone, C.-H. Lee et al., Flexible and transparent $\mathrm{MoS}_{2}$ field-effect transistors on hexagonal boron nitride-graphene heterostructures. ACS Nano 7(9), 7931-7936 (2013). doi:10.1021/nn402954e

212. S.-L. Li, K. Wakabayashi, Y. Xu, S. Nakaharai, K. Komatsu, W.-W. Li, Y.-F. Lin, A. Aparecido-Ferreira, K. Tsukagoshi, Thickness-dependent interfacial coulomb scattering in atomically thin field-effect transistors. Nano Lett. 13(8), 3546-3552 (2013). doi:10.1021/n14010783 
213. S.-L. Li, K. Tsukagoshi, Carrier injection and scattering in atomically thin chalcogenides. J. Phys. Soc. Jpn. 84(12), 121011 (2015). doi:10.7566/JPSJ.84.121011

214. X. Cui, G.-H. Lee, Y.D. Kim, G. Arefe, P.Y. Huang et al., Multiterminal transport measurements of $\mathrm{MoS}_{2}$ using a van der Waals heterostructure device platform. Nat. Nanotechnol. 10(6), 534-540 (2015). doi:10.1038/nnano.2015.70

215. K. Kaasbjerg, K.S. Thygesen, K.W. Jacobsen, Phonon-limited mobility in n-type single-layer $\mathrm{MoS}_{2}$ from first principles. Phys. Rev. B 85(11), 115317 (2012). doi:10.1103/PhysRevB.85.115317

216. B.W.H. Baugher, H.O.H. Churchill, Y. Yang, P. Jarillo-Herrero, Intrinsic electronic transport properties of high-quality monolayer and bilayer $\mathrm{MoS}_{2}$. Nano Lett. 13(9), 4212-4216 (2013). doi:10.1021/nl401916s

217. S. Larentis, B. Fallahazad, E. Tutuc, Field-effect transistors and intrinsic mobility in ultra-thin $\mathrm{MoSe}_{2}$ layers. Appl. Phys. Lett. 101(22), 223104 (2012). doi:10.1063/1.4768218

218. J.T. Ye, Y.J. Zhang, R. Akashi, M.S. Bahramy, R. Arita, Y. Iwasa, Superconducting dome in a gate-tuned band insulator. Science 338(6111), 1193-1196 (2012). doi:10.1126/Science1228006

219. B. Radisavljevic, A. Kis, Mobility engineering and a metalinsulator transition in monolayer $\mathrm{MoS}_{2}$. Nat. Mater. 12(9), 815-820 (2013). doi:10.1038/nmat3687

220. S.-L. Li, K. Komatsu, S. Nakaharai, Y.-F. Lin, M. Yamamoto, X. Duan, K. Tsukagoshi, Thickness scaling effect on interfacial barrier and electrical contact to two-dimensional $\mathrm{MoS}_{2}$ layers. ACS Nano 8(12), 12836-12842 (2014). doi:10.1021/nn506138y

221. S. M. Sze, K. K. Ng, Physics of Semiconductor Devices, 3rd edn. (Wiley, New Jersey, 2007)

222. S. Heinze, J. Tersoff, R. Martel, V. Derycke, J. Appenzeller, P. Avouris, Carbon nanotubes as Schottky barrier transistors. Phys. Rev. Lett. 89(10), 106801 (2002). doi:10.1103/PhysRevLett.89. 106801

223. Y. Guo, Y. Han, J. Li, A. Xiang, X. Wei, S. Gao, Q. Chen, Study on the resistance distribution at the contact between molybdenum disulfide and metals. ACS Nano 8(8), 7771-7779 (2014). doi:10.1021/nn503152r

224. A. Dankert, L. Langouche, M.V. Kamalakar, S.P. Dash, Highperformance molybdenum disulfide field-effect transistors with spin tunnel contacts. ACS Nano 8(1), 476-482 (2014). doi:10. 1021/nn404961e

225. J.-R. Chen, P.M. Odenthal, A.G. Swartz, G.C. Floyd, H. Wen, K.Y. Luo, R.K. Kawakami, Control of schottky barriers in single layer $\mathrm{MoS}_{2}$ transistors with ferromagnetic contacts. Nano Lett. 13(7), 3106-3110 (2013). doi:10.1021/n14010157

226. A. Gold, Electronic transport properties of a two-dimensional electron gas in a silicon quantum-well structure at low temperature. Phys. Rev. B 35(2), 723-733 (1987). doi:10.1103/Phys RevB.35.723

227. T. Ando, A.B. Fowler, F. Stern, Electronic properties of twodimensional systems. Rev. Mod. Phys. 54(2), 437-672 (1982). doi:10.1103/RevModPhys.54.437

228. S. Das Sarma, S. Adam, E.H. Hwang, E. Rossi, Electronic transport in two-dimensional graphene. Rev. Mod. Phys. 83(2), 407-470 (2011). doi:10.1103/RevModPhys.83.407

229. K. Kaasbjerg, K.S. Thygesen, A.-P. Jauho, Acoustic phonon limited mobility in two-dimensional semiconductors: deformation potential and piezoelectric scattering in monolayer $\mathrm{MoS}_{2}$ from first principles. Phys. Rev. B 87(23), 235312 (2013). doi:10.1103/PhysRevB.87.235312

230. N. Ma, D. Jena, Charge scattering and mobility in atomically thin semiconductors. Phys. Rev. X 4(1), 011043 (2014). doi:10. 1103/PhysRevX.4.011043

231. A.M. DaSilva, K. Zou, J.K. Jain, J. Zhu, Mechanism for current saturation and energy dissipation in graphene transistors. Phys.
Rev. Lett. 104(23), 236601 (2010). doi:10.1103/PhysRevLett. 104.236601

232. B.T. Moore, D.K. Ferry, Remote polar phonon scattering in $\mathrm{Si}$ inversion layers. J. Appl. Phys. 51(5), 2603-2605 (1980). doi:10.1063/1.327988

233. J. Hong, Z. Hu, M. Probert, K. Li, D. Lv et al., Exploring atomic defects in molybdenum disulphide monolayers. Nat. Commun. 6, 1-8 (2015). doi:10.1038/ncomms 7293

234. H. Schmidt, S. Wang, L. Chu, M. Toh, R. Kumar et al., Transport properties of monolayer $\mathrm{MoS}_{2}$ grown by chemical vapor deposition. Nano Lett. 14(4), 1909-1913 (2014). doi:10. $1021 / \mathrm{nl} 4046922$

235. E.H. Hwang, S. Adam, S.D. Sarma, Carrier transport in twodimensional graphene layers. Phys. Rev. Lett. 98(18), 186806 (2007). doi:10.1103/PhysRevLett.98.186806

236. X. Zou, Y. Liu, B.I. Yakobson, Predicting dislocations and grain boundaries in two-dimensional metal-disulfides from the first principles. Nano Lett. 13(1), 253-258 (2013). doi:10.1021/ n13040042

237. Y. Shi, H. Li, L.-J. Li, Recent advances in controlled synthesis of two-dimensional transition metal dichalcogenides via vapour deposition techniques. Chem. Soc. Rev. 44(9), 2744-2756 (2015). doi:10.1039/C4CS00256C

238. J. Yu, J. Li, W. Zhang, H. Chang, Synthesis of high quality twodimensional materials via chemical vapor deposition. Chem. Sci. 6(12), 6705-6716 (2015). doi:10.1039/C5SC01941A

239. W. Chen, J. Zhao, J. Zhang, L. Gu, Z. Yang et al., Oxygenassisted chemical vapor deposition growth of large single-crystal and high-quality monolayer $\mathrm{MoS}_{2}$. J. Am. Chem. Soc. 137(50), 15632-15635 (2015). doi:10.1021/jacs.5b10519

240. I. Bilgin, F. Liu, A. Vargas, A. Winchester, M.K.L. Man et al., Chemical vapor deposition synthesized atomically thin molybdenum disulfide with optoelectronic-grade crystalline quality. ACS Nano 9(9), 8822-8832 (2015). doi:10.1021/acsnano. 5b02019

241. Y. Yu, C. Li, Y. Liu, L. Su, Y. Zhang, L. Cao, Controlled scalable synthesis of uniform, high-quality monolayer and fewlayer $\mathrm{MoS}_{2}$ films. Sci. Rep. 3, 1-6 (2013). doi:10.1038/ srep01866

242. D.A. Bandurin, A.V. Tyurnina, G.L. Yu, A. Mishchenko, V. Zólyomi, S.V. Morozov et al., High electron mobility, quantum hall effect and anomalous optical response in atomically thin InSe. Nat. Nanotechnol. 12(3), 223-227 (2017). doi:10.1038/ nnano. 2016.242

243. H.C. Diaz, R. Addou, M. Batzill, Interface properties of CVD grown graphene transferred onto $\mathrm{MoS}_{2}$ (0001). Nanoscale 6(2), 1071-1078 (2014). doi:10.1039/c3nr03692h

244. N. Choudhary, J. Park, J.Y. Hwang, H.-S. Chung, K.H. Dumas, S.I. Khondaker, W. Choi, Y. Jung, Centimeter scale patterned growth of vertically stacked few layer only $2 \mathrm{D} \mathrm{MoS}_{2} / \mathrm{WS}_{2}$ van der Waals heterostructure. Sci. Rep. 6, 1-7 (2016). doi:10.1038/ srep25456

245. T. Roy, M. Tosun, J.S. Kang, A.B. Sachid, S.B. Desai, M. Hettick, C.C. Hu, A. Javey, Field-effect transistors built from all two-dimensional material components. ACS Nano 8(6), 6259-6264 (2014). doi:10.1021/nn501723y

246. R. Späh, M. Lux-Steiner, M. Obergfell, E. Bucher, S. Wagner, n- $\mathrm{MoSe}_{2} / \mathrm{p}-\mathrm{WSe}_{2}$ heterojunctions. Appl. Phys. Lett. 47(8), 871-873 (1985). doi:10.1063/1.95960

247. J.H. Yu, H.R. Lee, S.S. Hong, D. Kong, H.-W. Lee, H. Wang, F. Xiong, S. Wang, Y. Cui, Vertical heterostructure of two-dimensional $\mathrm{MoS}_{2}$ and $\mathrm{WSe}_{2}$ with vertically aligned layers. Nano Lett. 15(2), 1031-1035 (2015). doi:10.1021/nl503897h

248. M.-Y. Li, Y. Shi, C.-C. Cheng, L.-S. Lu, Y.-C. Lin et al., Epitaxial growth of a monolayer $\mathrm{WSe}_{2} / \mathrm{MoS}_{2}$ lateral $\mathrm{p}$-n junction 
with an atomically sharp interface. Science 349(6247), 524-528 (2015). doi:10.1126/Scienceaab4097

249. C.-H. Lee, G.-H. Lee, A.M. van der Zande, W. Chen, Y. Li et al., Atomically thin $\mathrm{p}-\mathrm{n}$ junctions with van der Waals heterointerfaces. Nat. Nanotechnol. 9(9), 676-681 (2014). doi: $10.1038 /$ nnano. 2014.150

250. A. Nourbakhsh, A. Zubair, M.S. Dresselhaus, T. Palacios, Transport properties of a $\mathrm{MoS}_{2} / \mathrm{WSe}_{2}$ heterojunction transistor and its potential for application. Nano Lett. 16(2), 1359-1366 (2016). doi:10.1021/acs.nanolett.5b04791

251. R. Cheng, D. Li, H. Zhou, C. Wang, A. Yin et al., Electroluminescence and photocurrent generation from atomically sharp $\mathrm{WSe}_{2} / \mathrm{MoS}_{2}$ heterojunction $\mathrm{p}-\mathrm{n}$ diodes. Nano Lett. 14(10), 5590-5597 (2014). doi:10.1021/n1502075n
252. D. Jariwala, V.K. Sangwan, C.-C. Wu, P.L. Prabhumirashi, M.L. Geier, T.J. Marks, L.J. Lauhon, M.C. Hersam, Gate-tunable carbon nanotube- $\mathrm{MoS}_{2}$ heterojunction p-n diode. Proc. Natl. Acad. Sci. 110(45), 18076-18080 (2013). doi:10.1073/pnas.1317226110

253. S. Chuang, R. Kapadia, H. Fang, T. Chia Chang, W.-C. Yen, Y.L. Chueh, A. Javey, Near-ideal electrical properties of InAs/ $\mathrm{WSe}_{2}$ van der Waals heterojunction diodes. Appl. Phys. Lett. 102(24), 242101 (2013). doi:10.1063/1.4809815

254. W. Zhang, Z. Huang, W. Zhang, Y. Li, Two-dimensional semiconductors with possible high room temperature mobility. Nano Res. 7(12), 1731-1737 (2014). doi:10.1007/s12274-0140532-X 